\title{
Downward Wave Coupling between the Stratosphere and Troposphere under Future Anthropogenic Climate Change ${ }^{\mathscr{O}}$
}

\author{
SANDRO W. LUBIS ${ }^{\mathrm{a}}$ \\ GEOMAR Helmholtz Centre for Ocean Research Kiel, Kiel, Germany \\ KATJA MATTHES \\ GEOMAR Helmholtz Centre for Ocean Research Kiel, and Christian-Albrechts-Universität zu Kiel, \\ Kiel, Germany \\ NILI HARNIK \\ Department of Geophysics, Tel Aviv University, Tel Aviv, Israel \\ NOUR-EDDINE OMRANI \\ Geophysical Institute, University of Bergen, and Bjerknes Centre for Climate Research, \\ Bergen, Norway \\ SEBASTIAN WAHL \\ GEOMAR Helmholtz Centre for Ocean Research Kiel, Kiel, Germany
}

(Manuscript received 5 June 2017, in final form 15 February 2018)

\begin{abstract}
Downward wave coupling (DWC) is an important process that characterizes the dynamical coupling between the stratosphere and troposphere via planetary wave reflection. A recent modeling study has indicated that natural forcing factors, including sea surface temperature (SST) variability and the quasi-biennial oscillation (QBO), influence DWC and the associated surface impact in the Northern Hemisphere (NH). In light of this, the authors further investigate how DWC in the NH is affected by anthropogenic forcings, using a fully coupled chemistry-climate model CESM1(WACCM). The results indicate that the occurrence of DWC is significantly suppressed in the future, starting later in the seasonal cycle, with more events concentrated in late winter (February and March). The future decrease in DWC events is associated with enhanced wave absorption in the stratosphere due to increased greenhouse gases (GHGs), which is manifest as more absorbing types of stratospheric sudden warmings (SSWs) in early winter. This early winter condition leads to a delay in the development of the upper-stratospheric reflecting surface, resulting in a shift in the seasonal cycle of DWC toward late winter in the future. The tropospheric responses to DWC events in the future exhibit different spatial patterns, compared to those of the past. In the North Atlantic sector, DWC-induced circulation changes are characterized by a poleward shift and an eastward extension of the tropospheric jet, while in the North Pacific sector, the circulation changes are characterized by a weakening of the tropospheric jet. These responses are consistent with a change in the pattern of DWC-induced synoptic-scale eddy-mean flow interaction in the future.
\end{abstract}

Supplemental information related to this paper is available at the Journals Online website: https://doi.org/10.1175/JCLI-D-17-0382.s1.

\footnotetext{
${ }^{\text {a }}$ Current affiliation: Department of the Geophysical Sciences, The University of Chicago, Chicago, Illinois

Corresponding author: Sandro W. Lubis, slubis@uchicago.edu
}

\section{Introduction}

Vertical propagation of planetary waves represents one of the most fundamental processes involved in the linkage between the tropospheric and stratospheric circulation. Planetary waves are generated in the troposphere by orographic and/or nonorographic forcing and propagate upward into the stratosphere, where either they 
break and induce downward-propagating zonal-mean wind anomalies (e.g., Kodera et al. 1990; Baldwin and Dunkerton 2001; Lubis et al. 2018), or they are reflected downward toward the troposphere (Perlwitz and Harnik 2003). The heat and momentum transports via planetary waves play an important role in controlling key aspects of middle- and high-latitude climate, including the distribution of temperature and ozone, midlatitude tropospheric jet, and stratospheric westerlies.

In recent years, evidence has accumulated that changes in the stratosphere can have a significant impact on the troposphere via downward planetary wave reflection from the stratosphere to the troposphere, known as downward wave coupling (DWC; e.g., Perlwitz and Harnik 2003; Shaw et al. 2010; Shaw and Perlwitz 2013; Lubis et al. 2016a, 2017). DWC events occur when upwardpropagating waves reach the stratosphere and then get reflected downward toward the troposphere, where they impact the wave and circulation there (Perlwitz and Harnik 2003; Shaw et al. 2010; Lubis et al. 2016a, 2017). Many episodes of DWC are tied to the so-called bounded wave geometry of the stratospheric basic state, which is characterized by a vertical reflecting surface in the upper stratosphere and a well-defined high-latitude meridional waveguide in the lower stratosphere (e.g., Harnik and Lindzen 2001; Shaw et al. 2010; Lubis et al. 2016a, 2017). Recent research has revealed that DWC has a significant impact on the tropospheric circulation and surface climate over the North Atlantic region during midwinter (Shaw and Perlwitz 2013; Shaw et al. 2014; Dunn-Sigouin and Shaw 2015; Lubis et al. 2016a). DWC signals in the troposphere resemble a positive phase of the North Atlantic Oscillation (NAO), characterized by a poleward tropospheric jet shift in the North Atlantic sector (Shaw and Perlwitz 2013; DunnSigouin and Shaw 2015; Lubis et al. 2016a). This tropospheric circulation change is intimately linked to a net acceleration of the polar vortex in the stratosphere, arising from the Eliassen-Palm (EP) flux divergence induced by DWC events (e.g., Dunn-Sigouin and Shaw 2015). More recently, Lubis et al. (2016a) showed that the tropospheric response to DWC is dominated by eddy-mean flow feedbacks, which are excited by the initial downward wave reflection in the stratosphere. In particular, following the wave- 1 reflection in the stratosphere, a wave-1 geopotential height anomaly-like pattern emerges in the high-latitude troposphere. This anomaly gives rise to increased winds in the high-latitude North Atlantic sector, as indicated by a poleward shift of the tropospheric jet, and an anomalous positive NAOlike response. This positive NAO-like response is further strengthened by synoptic-scale eddy feedback due to changes in lower-level baroclinicity induced by increased vertical wind shear and SST forcing. Thus, a better knowledge of DWC and the involved mechanisms will help to improve the representation of tropospheric circulation and surface climate in climate models.

The influence of future anthropogenic climate change on the Northern Hemisphere (NH) winter stratosphere has been discussed in great detail in model studies using twenty-first-century greenhouse gas (GHG) emission scenarios (e.g., Charlton-Perez et al. 2008; Ayarzagüena et al. 2013; Manzini et al. 2014). Under the representative concentration pathway 8.5 (RCP8.5) scenario, Manzini et al. (2014) showed that the majority of CMIP5 models predict a weaker stratospheric zonalmean wind at high latitudes in the $\mathrm{NH}$ winter. This result is supported by the majority of general circulation model (GCM) studies that show an increase in the frequency of stratospheric sudden warming (SSW) in response to increased GHG forcing (e.g., Butchart et al. 2000; Charlton-Perez et al. 2008; Bell et al. 2010; Ayarzagüena et al. 2013; Schimanke et al. 2013). One of the possible mechanisms that leads to such an increase is the upward shift in the location of critical layers, which leads to more waves penetrating and converging into the subtropical lower stratosphere due to the strengthening of the upper flanks of the subtropical jet (Shepherd and McLandress 2011). Other mechanisms, based on idealized model simulations, show that an increased energy cascade from the organization of baroclinic eddies (Tung and Orlando 2003) would cause an enhanced upward propagation of large-scale planetary waves into the subtropical stratosphere (Eichelberger and Hartmann 2005). Other studies using atmospheric chemistry-climate models (CCMs) (Oberländer et al. 2013; Ayarzagüena et al. 2013) argue that a deepening of the Aleutian low in response to climate change could also lead to enhanced upward wave propagation into the stratosphere through positive interference of wave activity. The aforementioned studies have thus demonstrated a range of mechanisms by which upwardpropagating waves lead to a weakening of the polar vortex under GHG-induced climate change. However, the effect of DWC on circulation in the stratosphere and troposphere under future climate change in the $\mathrm{NH}$ remains unknown. In this study, we extend the investigations by using a stateof-the-art chemistry-climate model, the Community Earth System Model, version 1 (Whole Atmosphere Community Climate Model) [CESM1(WACCM)], which has both a fully resolved stratosphere and a fully coupled ocean. In this way, the significance of coupled ocean feedbacks in, for example, generating ocean-land contrasts and shaping the tropospheric response to DWC, as well as the importance of atmospheric chemistry for vortex variability, are included. 
Using a set of sensitivity simulations with CESM1(WACCM), consisting of a number of single natural forcing experiments [i.e., anthropogenic GHGs and ozone depleting substances (ODSs) are kept constant at 1960s levels], Lubis et al. (2016a) showed that natural forcing factors, including SST and the quasi-biennial oscillation (QBO), are equally important in establishing a correct representation of DWC in the CCM. Excluding SST (QBO) forcing caused the DWC frequency to drop (increase) significantly. In addition, the QBO and SST variability also influence the tropospheric response to DWC, both through a modification of wave propagation and interaction with the mean flow in the stratosphere and through a modification of the synoptic-scale eddy-mean flow feedbacks, which are excited by the initial downward wave reflection (Lubis et al. 2016a). On the other hand, the role of anthropogenic forcing factors, including GHGs and ODSs on DWC, has so far only been examined in the Southern Hemisphere (SH) (Shaw et al. 2011). Shaw et al. (2011) showed that a significantly increased DWC in the SH spring in the period of past ozone depletion can be attributed mainly to increased anthropogenic ODSs, while there is no significant change in the occurrence of DWC events in response to future GHG forcing. Despite these results, the importance of the anthropogenic forcing factors on DWC in the $\mathrm{NH}$ remains unknown and will be addressed within this study.

The goal of the present study is to investigate the impact of future anthropogenic climate change on DWC events in the $\mathrm{NH}$ winter stratosphere, particularly how their seasonality will change in the future and how different anthropogenic forcings (GHG and ODSs) individually influence the occurrence of these events. We focus only on total planetary waves with zonal wavenumber 1 , since it is the dominant source of DWC in the NH (Perlwitz and Harnik 2003). In addition, we also examine how these anthropogenic forcings can affect the downward influence of DWC on troposphere-surface climate in the future. To this end, we use different transient and timeslice simulations with a fully coupled chemistry-climate model [CESM1(WACCM)] to investigate the impact of anthropogenic climate change on DWC and the underlying mechanisms. A description of the data, model experiments, and method is given in section 2 . Section 3 describes the influence of future anthropogenic climate change on the background states, wavemean flow interaction, and DWC. In section 4, we assess the impact of DWC on future tropospheresurface climate over the North Atlantic and North
Pacific sectors. The paper concludes with a summary and discussion in section 5 .

\section{Model, experiments, and methods}

\section{a. Model and experimental details}

All simulations used in this study were performed within the National Center for Atmospheric Research (NCAR) Community Earth System Model version 1.0.2, a fully coupled global Earth system model, which contains interactive ocean, land, sea ice, and atmosphere components (Gent et al. 2011; Hurrell et al. 2013). The Whole Atmosphere Community Climate Model version 4 (Marsh et al. 2013) is used for the atmosphere component, with 66 standard vertical levels (up to $5.1 \times 10^{-6} \mathrm{hPa}$ or $\sim 140 \mathrm{~km}$ ) and a horizontal resolution of $1.9^{\circ}$ latitude $\times$ $2.5^{\circ}$ longitude. The model is coupled with interactive atmospheric chemistry, which is calculated within the 3D chemical transport Model of Ozone and Related Chemical Tracers, version 3 (MOZART-3; Kinnison et al. 2007). The model includes a total of 59 species, such as $\mathrm{O}_{x}, \mathrm{NO}_{x}, \mathrm{HO}_{x}, \mathrm{ClO}_{x}, \mathrm{BrO}_{x}$, and $\mathrm{CH}_{4}$, and 217 gas-phase chemical reactions (Marsh et al. 2013). The interactive radiation and chemistry are implemented from the surface up to the lower thermosphere so that some important processes in the middle atmosphere, such as ion chemistry, auroral processes, and nonlocal thermodynamic equilibrium radiation, are simulated (Marsh et al. 2013).

To investigate the influence of anthropogenic climate change on Northern Hemisphere DWC between the stratosphere and troposphere, we performed one longterm transient (TR) simulation with varying radiative forcings, covering the period from 1955 to 2099 (145 years; Table 1). This simulation is forced with GHGs and ODSs following observations until 2005 and the RCP8.5 scenario ${ }^{1}$ (Meinshausen et al. 2011) out to the year 2100 (hereafter referred to as the TR-RCP8.5 run). This simulation includes a representation of the QBO, implemented by relaxing equatorial zonal winds between $22^{\circ} \mathrm{S}$ and $22^{\circ} \mathrm{N}$ toward observations, following Matthes et al. (2010) and extended into the future by projecting Fourier coefficients of the oscillation. ${ }^{2}$ The solar spectral irradiance is specified as spectrally resolved daily variations obtained from the model of Lean et al. (2005). This simulation is run with interactive ocean and

\footnotetext{
${ }^{1}$ The radiative forcing reaches a maximum of $\sim 8.5 \mathrm{~W} \mathrm{~m}^{-2}$ in 2100 .

${ }^{2}$ The QBO is projected into the future by developing Fourier coefficients for the QBO time series based on climatological values of Giorgetta (http://www.pa.op.dlr.de/CCMVal/Forcings/qbo_data_ccmval/ u_profile_195301-200412.html) from the past records (1954-2004).
} 
TABLE 1. Description of CESM1(WACCM) TR and TS experiments. All experiments are run with QBO nudging and with interactive chemistry and SSTs/sea ice.

\begin{tabular}{lclc}
\hline \hline Experiment & Period & GHG & ODS \\
\hline CTRL & $1955-2099(145$ years $)$ & Fixed at 1960s level & Fixed at 1960s level \\
TR-RCP8.5 & $1955-2099(145$ years $)$ & Obs + RCP8.5 & Obs + RCP8.5 \\
TS-ODS & 40 years & Fixed at 2080 level & Fixed at 1960s level \\
TS-GHG & 40 years & Fixed at 1960s level & Fixed at 2080s level \\
\hline
\end{tabular}

${ }^{a}$ GHG/ODS follows observations until 2005 and the RCP8.5 scenario thereafter.

sea ice. In addition, a 145-yr control simulation (hereafter referred to as the CTRL run) is also used, in which the model is run with fixed GHGs and ODSs at 1960s levels (i.e., no varying radiative forcing over the whole simulation period) so that the internal variability may be estimated. All other settings are equivalent to the TR-RCP8.5 simulation. Both model simulations (TRRCP8.5 and CTRL) are initialized using initial files for January 1955 from a CESM-piControl experiment ${ }^{3}$ from the CESM contribution to CMIP5, which runs for several hundred years to reach an equilibrium state in the ocean. Future changes in DWC characteristics are assessed by comparing the last 40 winters of TR-RCP8.5 (2060-99, "future") with the first 40 winters (1960-99, "past").

We also employ different timeslice (TS) simulations of about 40 years with the same model, which include separate changes in concentrations in GHG or ODS for present and projected future climates. TS simulations are climate model experiments that repeat all or most external forcings for a specific year, while others follow an observed or projected record (e.g., Ayarzagüena et al. 2013; Lubis et al., 2016b). In our setup, the TSGHG experiment uses seasonally varying surface emissions of ODSs at 1960s levels in combination with surface emissions of GHGs at 2080s levels. As for the TS-ODS experiment, ODSs at 2080s levels in combination with surface emissions of GHGs at 1960s levels are used. All TS experiments are initialized using the background state from year 2080 of the TR-RCP8.5 run. All other external forcings (e.g., aerosols, $\mathrm{NO}_{2}$ aircraft emissions) are averaged \pm 5 years around 2080 for both TS experiments. These sensitivity simulations allow us to isolate the influence of each anthropogenic forcing (GHG and ODS) on DWC. A detailed description of each TR and TS simulation is provided in Table 1.

\footnotetext{
${ }^{3}$ See http://www.cgd.ucar.edu/ccr/strandwg/CMIP5_experiment_ list.html.
}

\section{b. Wave diagnostics}

We use a time-lagged singular value decomposition (SVD) analysis to separate upward- and downwardpropagating planetary wave signals between the stratosphere and troposphere (Perlwitz and Harnik 2003; Lubis et al. 2016a). This diagnostic isolates the leading coupled modes that represent the maximum covariance between two daily geopotential heights of zonal wavenumber $k$ at two pressure levels $(500$ and $10 \mathrm{hPa})$ for each time lag $\tau$ separately. The maximum relationship between the two wave fields is determined by the correlation of temporal expansion coefficients $(A$ and $B)$ of the leading coupled mode $\left[A^{k}(t), B^{k}(t+\tau)\right]$. The daily temporal expansion coefficients are calculated following Bretherton et al. (1992), in which data at each grid point are linearly projected onto its corresponding EOFs as

$$
\begin{aligned}
A^{k}(t) & =\sum_{i=1}^{M_{p}} V_{i}^{k} P_{i}(t)=\mathbf{V}_{k}^{\mathrm{T}} P(t) \quad \text { and } \\
B^{k}(t+\tau) & =\sum_{j=1}^{M_{s}} U_{j}^{k} S_{j}(t+\tau)=\mathbf{U}_{k}^{\mathrm{T}} S(t+\tau),
\end{aligned}
$$

where $P$ and $S$ signify daily tropospheric and stratospheric geopotential heights of zonal wavenumber $k$, respectively, and $M$ indicates the number of grid points. The left and right singular vectors at mode $k$ are denoted by $\mathbf{V}_{k}$ and $\mathbf{U}_{k}$, respectively. We choose $500 \mathrm{hPa}$ as a reference level so that the upward- (downward) propagating wave is identified when the wave correlations are statistically significant at the positive (negative) time lags. Here, we are interested in the zonal wavenumber 1 because it is the dominant source of DWC in the $\mathrm{NH}$ (Perlwitz and Harnik 2003). We repeat the diagnostic for the entire seasons with 3-month overlapping periods, as in Lubis et al. (2016a).

In addition, a diagnostic of the basic-state wave propagation characteristics (Harnik and Lindzen 2001; Lubis et al. 2016a, 2017) is used to determine the existence and location of reflecting surfaces for meridional and vertical wave propagation. This diagnostic is a more accurate indicator of wave propagation regions (rather than the 
index of refraction), since it diagnoses meridional and vertical propagation separately. For a nonisothermal atmosphere, the wavenumbers are diagnosed from the solution to the Rossby wave equation associated with the quasi-geostrophic (QG) conservation of potential vorticity (PV; Harnik and Lindzen 2001) (presented here for illustrative purposes in Cartesian coordinates):

$$
\frac{\partial^{2} \psi}{\partial z^{2}}+\frac{N^{2}}{f^{2}} \frac{\partial^{2} \psi}{\partial y^{2}}+n_{r}^{2} \psi=0,
$$

where $\psi=\Phi / 2 \Omega \sin \phi$ is the geopotential streamfunction, $\Phi$ is geopotential, $\Omega$ is the rotation rate of the planet, $N^{2}$ is the Brunt-Väisälä frequency, $f$ is the Coriolis parameter, and $n_{r}^{2}$ is as follows:

$$
\begin{aligned}
n_{r}^{2} & \equiv \frac{N^{2}}{f^{2}}\left\{\frac{\bar{q}_{y}}{\bar{u}-c}-k^{2}+f^{2} \frac{2^{z / 2 H}}{N} \frac{\partial}{\partial z}\left[\frac{e^{-z / H}}{N^{2}} \frac{\partial}{\partial z}\left(e^{z / 2 H} N\right)\right]\right\} \\
& \equiv m^{2}+\frac{N^{2}}{f^{2}} l^{2} .
\end{aligned}
$$

Here, $\bar{u}$ is zonal-mean wind, $\bar{q}_{y}$ is the meridional gradient of zonal-mean $\mathrm{PV}, H$ is scale height, and $k$ and $c$ are the zonal wavenumber and phase speeds, respectively. We focus on zonal wavenumber 1 and set $c$ to zero so that we consider only stationary wavenumber 1 . The coefficients of the wave in Eq. (3) are calculated using monthly mean zonal-mean zonal wind and temperature data. The vertical and meridional wavenumbers are subsequently diagnosed from the solution to the wave equation as $m^{2}=-\operatorname{Re}\left(\psi_{z z} / \psi\right)$ and $l^{2}=-\operatorname{Re}\left(\psi_{y y} / \psi\right)$, respectively [see Harnik and Lindzen (2001) for detailed theoretical considerations]. A vertical reflecting surface for vertical wave propagation is the $m^{2}=0$ surface.

We also quantify the contribution of 3D planetaryscale wave flux (represented by $\mathbf{F}_{s}$ vectors; Plumb 1985; see also the appendix) and 3D synoptic (transient) wave flux (represented by $\mathbf{E}$ vectors; Hoskins et al. 1983) on the mean flow. The 3D synoptic (transient) wave flux vectors $\mathbf{E}$ roughly point in the direction of the synoptic (baroclinic) wave energy propagation, and its convergence indicates a deceleration of the zonal flow due to baroclinic wave forcing. The 3D synoptic-scale wave activities are computed as follows:

$$
\mathbf{E}=\left\{\begin{array}{c}
\overline{u^{\prime 2}-v^{\prime 2}} \\
-\overline{v^{\prime} u^{\prime}} \\
-f\left(\frac{\partial \theta}{\partial p}\right)^{-1} \overline{v^{\prime} \theta^{\prime}}
\end{array}\right\},
$$

where $v, \theta$, and $p$ are the meridional wind, potential temperature, and pressure level, respectively. The prime in $\mathbf{E}$ vectors denotes a 2-6-day bandpass Butterworthfiltered daily anomaly, which represents the highfrequency baroclinic wave activity (Blackmon 1976). The overbar signifies a time average. In addition, the upper-level storm-track activity is also analyzed and is calculated as the variance of $200-\mathrm{hPa}$ meridional wind $\overline{v^{\prime} v^{\prime}}$, which represents eddy activity aloft during a mature stage of the baroclinic eddy life cycle when perturbations are well developed (Wettstein and Wallace 2010).

\section{c. Individual DWC event definition}

An individual DWC event is identified based on the daily total negative wave-1 meridional heat flux $\overline{v^{\prime} T^{\prime}}{ }_{k=1}$ at $50 \mathrm{hPa}$, weighted by the cosine of latitude and meridionally averaged between $60^{\circ}$ and $90^{\circ} \mathrm{N}$ (DunnSigouin and Shaw 2015; Lubis et al. 2016a, 2017). The DWC event is defined when the ${\overline{v^{\prime} T^{\prime}}}_{k=1}$ at $50 \mathrm{hPa}$ series drops below the 5th percentile of the January-March (JFM) distribution. The central date (day 0 ) is defined as the day of minimum $\overline{v^{\prime} T^{\prime}}{ }_{k=1}$, and each event must be separated by at least 15 days. This time separation is motivated by the time scale of planetary wave coupling between the stratosphere and troposphere (Perlwitz and Harnik 2003). The $\overline{v^{\prime} T^{\prime}}{ }_{k=1}$ is often negative after SSW events (Kodera et al. 2016), and such a type of reflection is closely related to wave overreflection [see Tomikawa (2010) for a detailed discussion]. Therefore, in order to ensure that we only examine DWC events, we exclude from the DWC date event found above those for which an SSW occurs within its duration or within 3-10 days after the onset of SSW events.

Qualitatively similar results are obtained for different choices of the reference level (e.g., $\overline{v^{\prime} T^{\prime}}{ }_{k=1}$ at 30 and $10 \mathrm{hPa}$ ) or time separation. The statistical significance of the DWC's life cycle composites is calculated by performing a 1000-trial Monte Carlo analysis, following Lubis et al. (2017). The anomalies for the composites are defined as the deviations from the daily climatological seasonal cycle.

\section{Effect of climate change on DWC}

In this section, the impact of future anthropogenic climate change on DWC is presented by first discussing the impact on the temperature, background wind, and wave-mean flow interaction. Then, we diagnose the respective impacts on DWC by analyzing the wave coupling correlation and seasonal variation in wave geometries.

\section{a. Stratospheric basic state responses}

It is well established that the stratospheric basic states determine the transmission or refraction properties of 

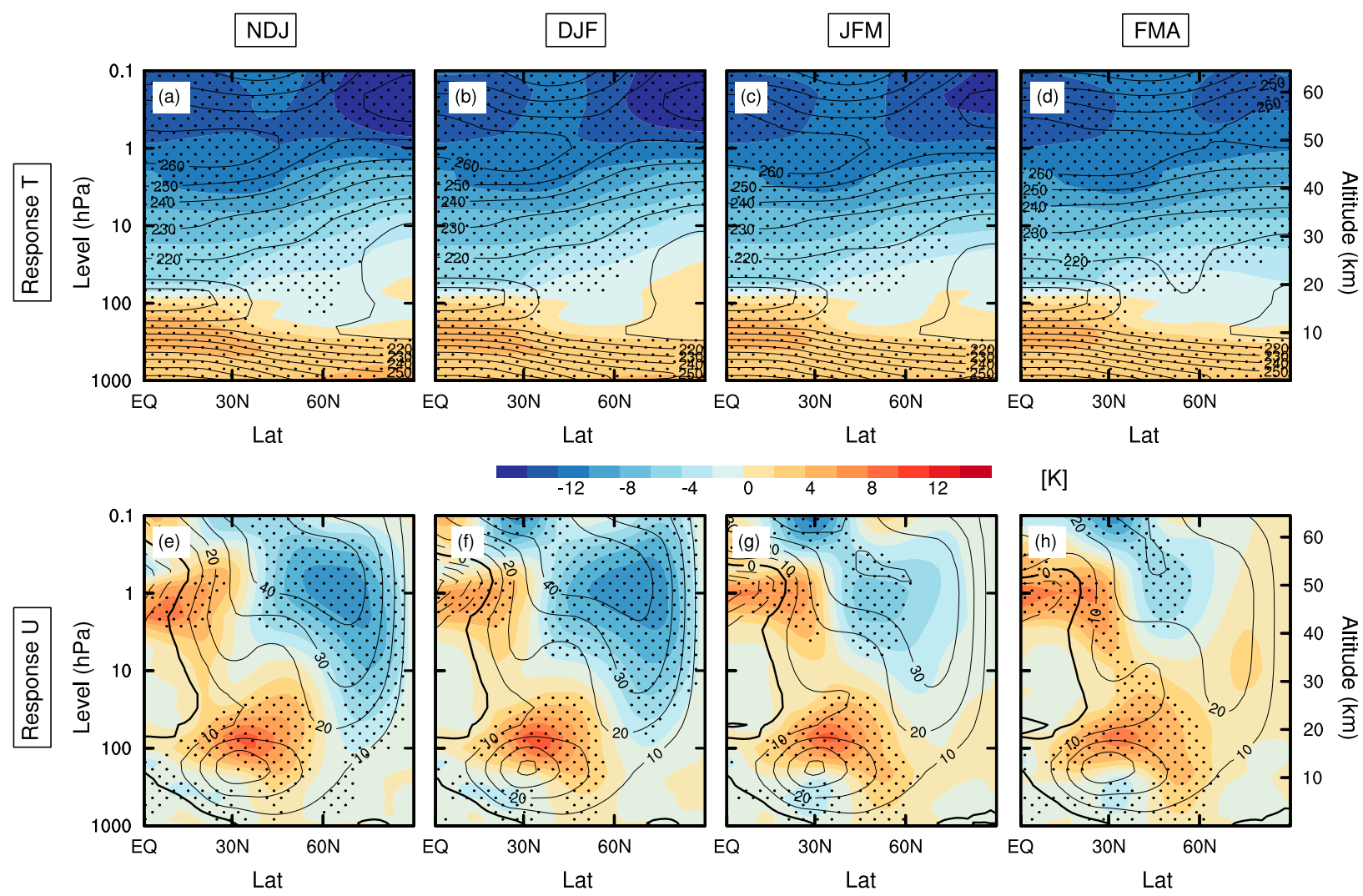

$[\mathrm{K}]$
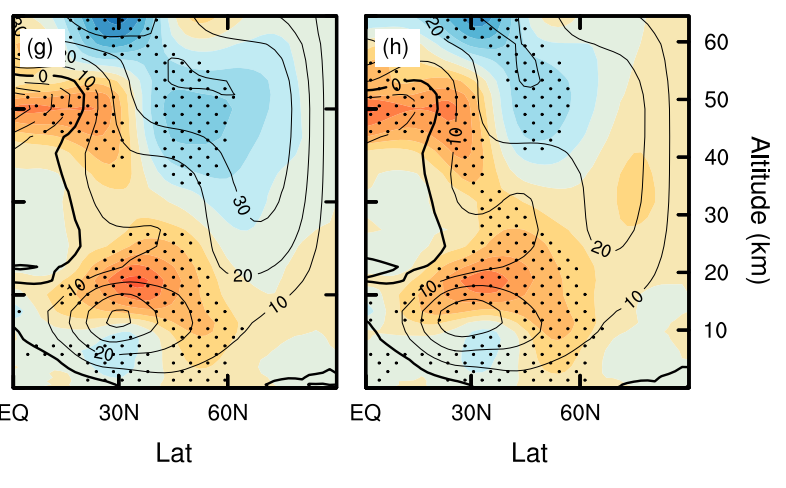

$\begin{array}{lllllllllllllllll}-8 & -7 & -6 & -5 & -4 & -3 & -2 & -1 & 0 & 1 & 2 & 3 & 4 & 5 & 6 & 7 & 8\end{array} \quad[\mathrm{~m} / \mathrm{s}]$

FIG. 1. Differences in the zonal-mean (top) temperature and (bottom) wind between the past (1960-99) and future (2060-99) climatologies for the TR-RCP8.5 run during (a),(e) NDJ, (b),(f) DJF, (c),(g) JFM, and (d),(h) FMA. The black contour lines indicate the climatology from the CTRL run. The temperature responses use contour intervals of $2 \mathrm{~K}$; for the zonal wind responses, the contour interval is $1 \mathrm{~m} \mathrm{~s}^{-1}$. Contour intervals from the CTRL are $10 \mathrm{~K}$ and $10 \mathrm{~m} \mathrm{~s}^{-1}$ for the temperature and zonal wind climatologies, respectively. Dotted areas indicate regions where the signal is statistically significant at the $95 \%$ level, according to a two-tailed $t$ test.

vertically propagating planetary waves (Charney and Drazin 1961; Matsuno 1970). In turn, the changes in the behavior of planetary waves can affect the basic states. Therefore, it is important to first examine how the temperature, background wind, and propagation properties of planetary waves are changing in response to future anthropogenic climate change.

Figure 1 shows the zonal-mean temperature and zonal wind differences in the transient run between 40 winters in the recent past (1960-99) and 40 winters at the end of the twenty-first century (2060-99), which give a measure of the atmospheric response to an increase in GHG. We note that by the end of the twenty-first century, ozone concentration has recovered to preozone hole levels (Lubis et al. 2016b) so that the differences in the stratospheric response by this time can be primarily attributed to increased GHG levels. The change in stratospheric temperatures over the twenty-first century is characterized by a globally averaged stratospheric cooling (with the magnitude of changes up to $12 \mathrm{~K}$ ) and tropospheric heating (up to $5 \mathrm{~K}$ ) (Figs. 1a-d). The maximum cooling takes place from November to January (NDJ) and is situated near the stratopause at $1 \mathrm{hPa}$, where the stratospheric temperatures are highest. In addition, certain areas in the polar lower stratosphere are warmer (especially in DJF), which is consistent with increased SSW events in the future (not shown). However, the signal is not significant, which is likely due to high levels of variability in the polar northern latitudes (e.g., due to the presence of SSWs) (Mitchell et al. 2012; Hansen et al. 2014). Bell et al. (2010) found that it was not the case for the idealized scenario of 4 times preindustrial $\mathrm{CO}_{2}$, where the results become significant at these latitudes. The corresponding plot for the zonal winds (Figs. 1e,f) shows a deceleration of the stratospheric polar winds (up to $5 \mathrm{~m} \mathrm{~s}^{-1}$ ), suggesting a more disturbed polar vortex. The maximum deceleration occurs from early winter to midwinter (NDJ), with a magnitude of up to $5 \mathrm{~m} \mathrm{~s}^{-1}$, and gradually shifts upward and loses significance from February to April (FMA). In the troposphere, there 

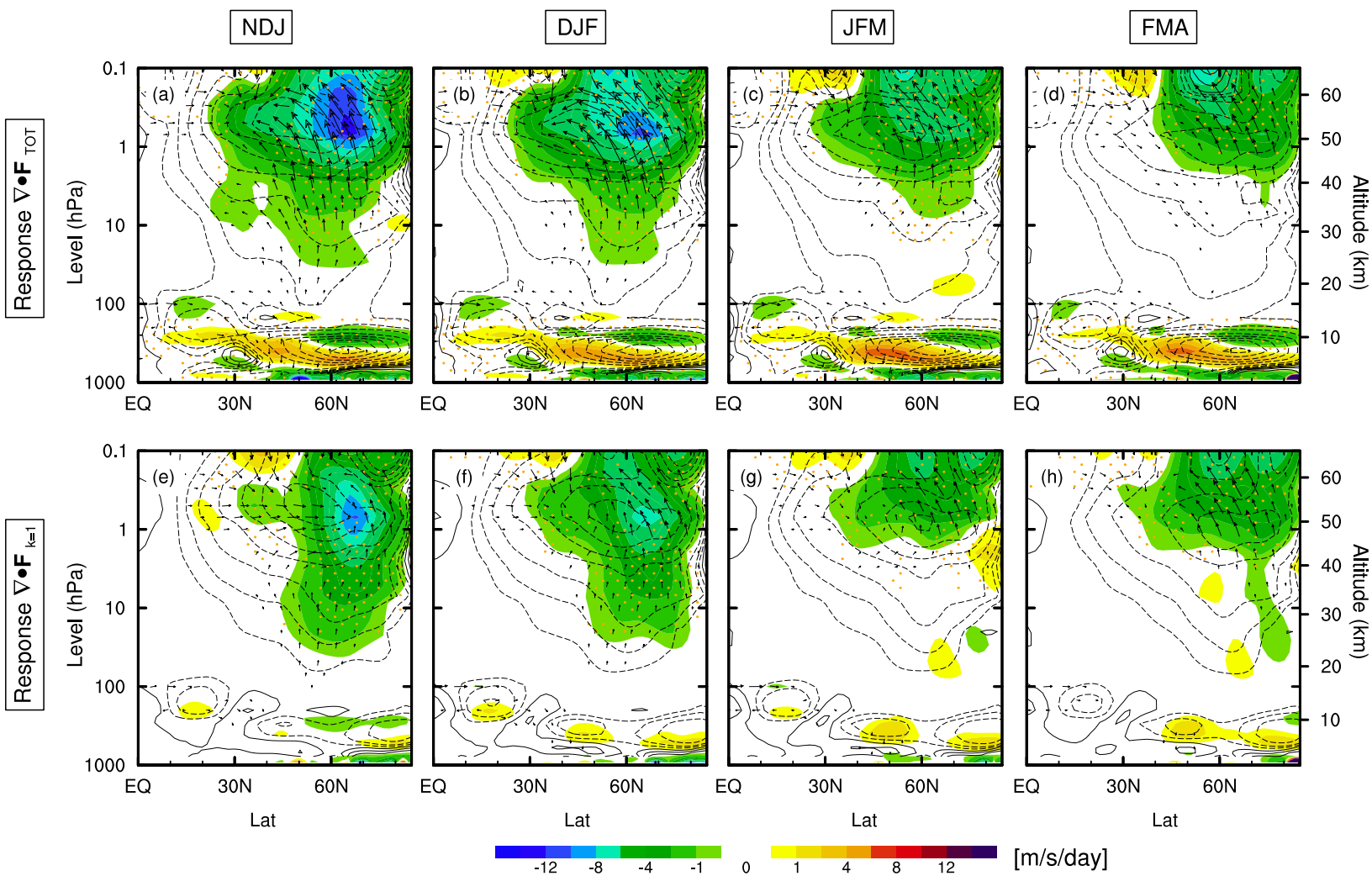

FIG. 2. Differences in the (top) total and (bottom) wave-1 EP flux vectors between the past (1960-99) and future (2060-99) climatologies, as well as the corresponding differences in EP flux divergence (shadings), from the TR-RCP8.5 run during (a),(e) NDJ, (b),(f) DJF, (c),(g) JFM, and (d),(h) FMA. The black contour lines indicate the climatology of EP flux divergence from the CTRL run. The contour intervals are in logarithmic powers of $2: \pm(0.5,1,2,4,8,16,32,64, \ldots) \mathrm{m} \mathrm{s}^{-1}$ day $^{-1}$. Dotted areas indicate regions where the signal is statistically significant at the $95 \%$ level, according to a two-tailed $t$ test.

is a poleward and upward shift of the tropospheric jet in response to increases in GHGs across all seasons from NJF to FMA. These results are similar to most previous CCM studies using the RCP8.5 scenario and CMIP5 results (e.g., Mitchell et al. 2012; Ayarzagüena et al. 2013; Schmidt et al. 2013), although the peak of the maximum wind deceleration in the stratosphere from the previous studies occurred somewhat late in midwinter, from January to March. A possible reason for this discrepancy might be due to the competition of different contributors and the biases of each model to produce the correct dynamical responses for the interaction between the stratosphere and GHGs or ozone changes (SPARC CCMVal 2010). The weakening of the polar vortex in response to future climate change would suggest an increase in wave absorption and a reduction in the downward wave reflection in the stratosphere.

\section{b. Wave-mean flow interaction responses}

Figure 2 shows the 3-month running mean differences of the EP flux vector and the associated divergence. The EP flux vector is a measure for the direction of planetary wave propagation, and its divergence indicates the tendency of the zonal-mean flow in response to eddy forcing. From NDJ to DJF (Figs. 2a,b), there is a strong difference in the EP flux at high latitudes (i.e., more upward propagation of planetary waves from the troposphere in the future) from the lower to the upper stratosphere. Therefore, more wave dissipation or absorption at high latitudes leads to a significant deceleration of the stratospheric polar night jet (Figs. 1e,f). The EP flux convergence anomalies in DJF are larger compared to NDJ, which is consistent with stronger stratospheric wind deceleration in DJF. Planetary waves propagating from the troposphere upward into the stratosphere become weaker in JFM, with significant convergence anomalies mainly situated in the upper stratosphere and lower mesosphere (Fig. 2c). This behavior is consistent with significant easterly wind anomalies in the upper stratosphere and the equatorward shift of the easterly wind anomalies in the lower mesosphere in JFM (Fig. 1g).

The shift in the EP flux convergence anomalies continues to evolve in late winter (Fig. 2d), but with significant values concentrated above $40 \mathrm{~km}$. This is 
(a) TR-RCP8.5: (Past)

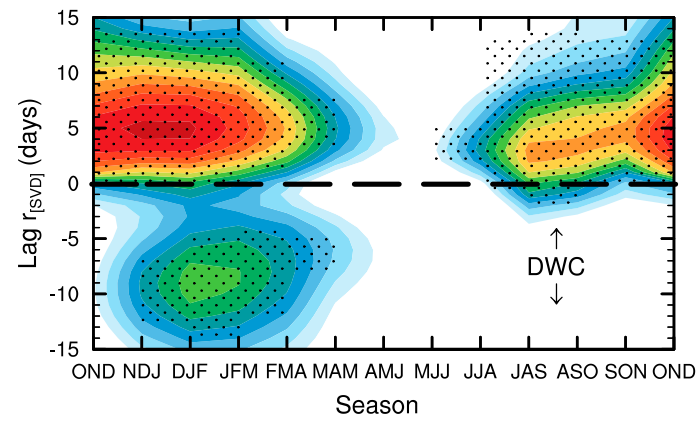

(c) TS-ODS: (Future)

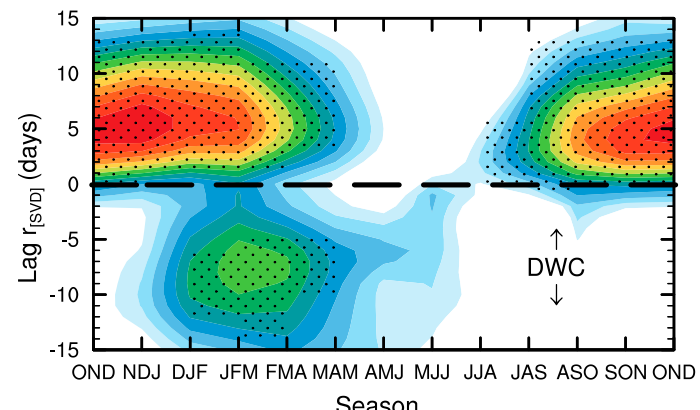

Season (b) TR-RCP8.5: (Future)

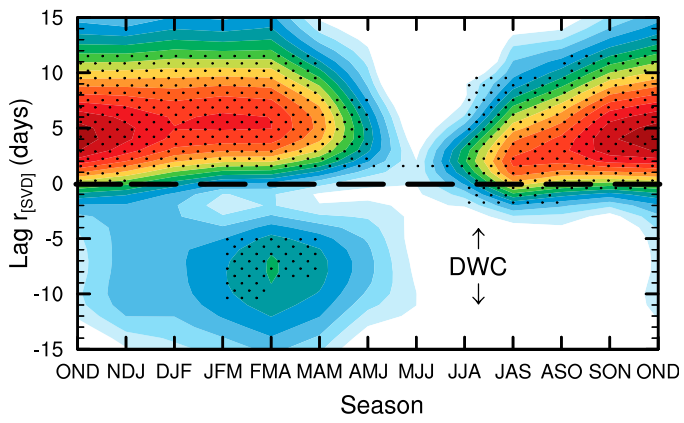

(d) TS-GHG: (Future)

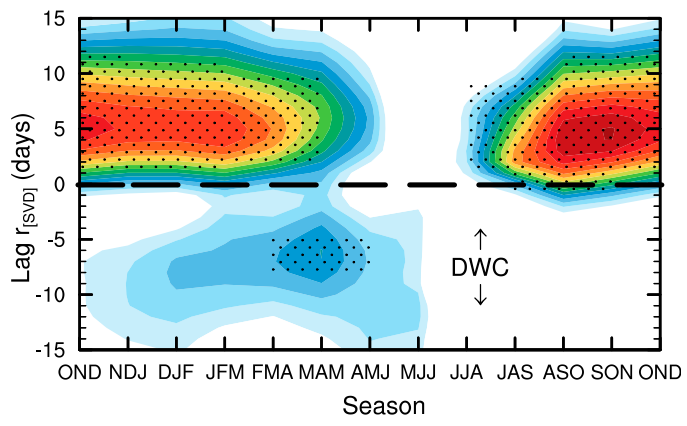

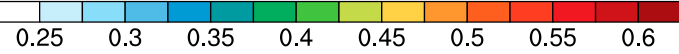

FIG. 3. Three-month overlapping periods of lagged SVD correlations between wave-1 geopotential height Z-ZWN1 at 500 and $10 \mathrm{hPa}$ for (a) TR-RCP8.5 past (1960-99), (b) TR-RCP8.5 future (2060-99), and two TS experiments with future (c) ODS and (d) GHG forcings. Solid dots represent values significant at the $99 \%$ level. A negative (positive) time lag indicates that the stratospheric (tropospheric) wave field is leading.

consistent with upward and equatorward shifts of easterly wind anomalies into the upper stratosphere in late winter (Fig. 1h). Furthermore, Figs. 2e-h show the differences of the zonal wave-1 EP flux vector and its divergence from early winter to late winter. It can be seen that both the pattern and magnitude of EP flux convergence from the total eddies (Figs. 2a-d) are, to a large degree, attributed to the wave- 1 convergence anomalies (Figs. 2e-h). We also note that the high-latitude wave-1 EP flux convergence is dominated by the vertical component (not shown).

In summary, the changes in EP flux convergence from early to late winter are consistent with the magnitude of deceleration of the $\mathrm{NH}$ vortex winds in the future, which is strongest in early winter. This behavior may suggest a transition from stronger wave absorption in early winter to a weaker wave absorption in late winter in the future. We will discuss this implication on DWC further in the following section.

\section{c. Seasonality of DWC events}

We now analyze the impact of future climate change on the timing in the seasonal cycle of DWC by first examining the wave coupling correlation and then the evolution of the wave geometry. Figure 3 shows 3 -month overlapping periods of lagged SVD correlations (rSVD) between geopotential heights of zonal wavenumber 1 (Z-ZWN1) at 500 and $10 \mathrm{hPa}$. Positive lags indicate upward wave propagation from the troposphere to the stratosphere, whereas negative lags indicate downward wave propagation (associated with wave reflection) from the stratosphere to the troposphere. These events are only considered if the signals are statistically significant at the 99\% level (Perlwitz and Harnik 2003; Lubis et al. 2016a). In the recent past, there is significant downward wave propagation throughout the extended winter, as indicated by significant correlations at negative time lags from November to March (Fig. 3a). This period is somewhat longer compared to the observation, which mostly occurs from January to March (e.g., Shaw et al. 2010; Lubis et al. 2016a). The downward wave activity maximizes at about 6-12 days from DJF to JFM. The time scales of downward propagation are also longer compared to the observations (e.g., Shaw et al. 2010; Lubis et al. 2016a), suggesting a slower downward group velocity of Z-ZWN1 from the stratosphere to the 

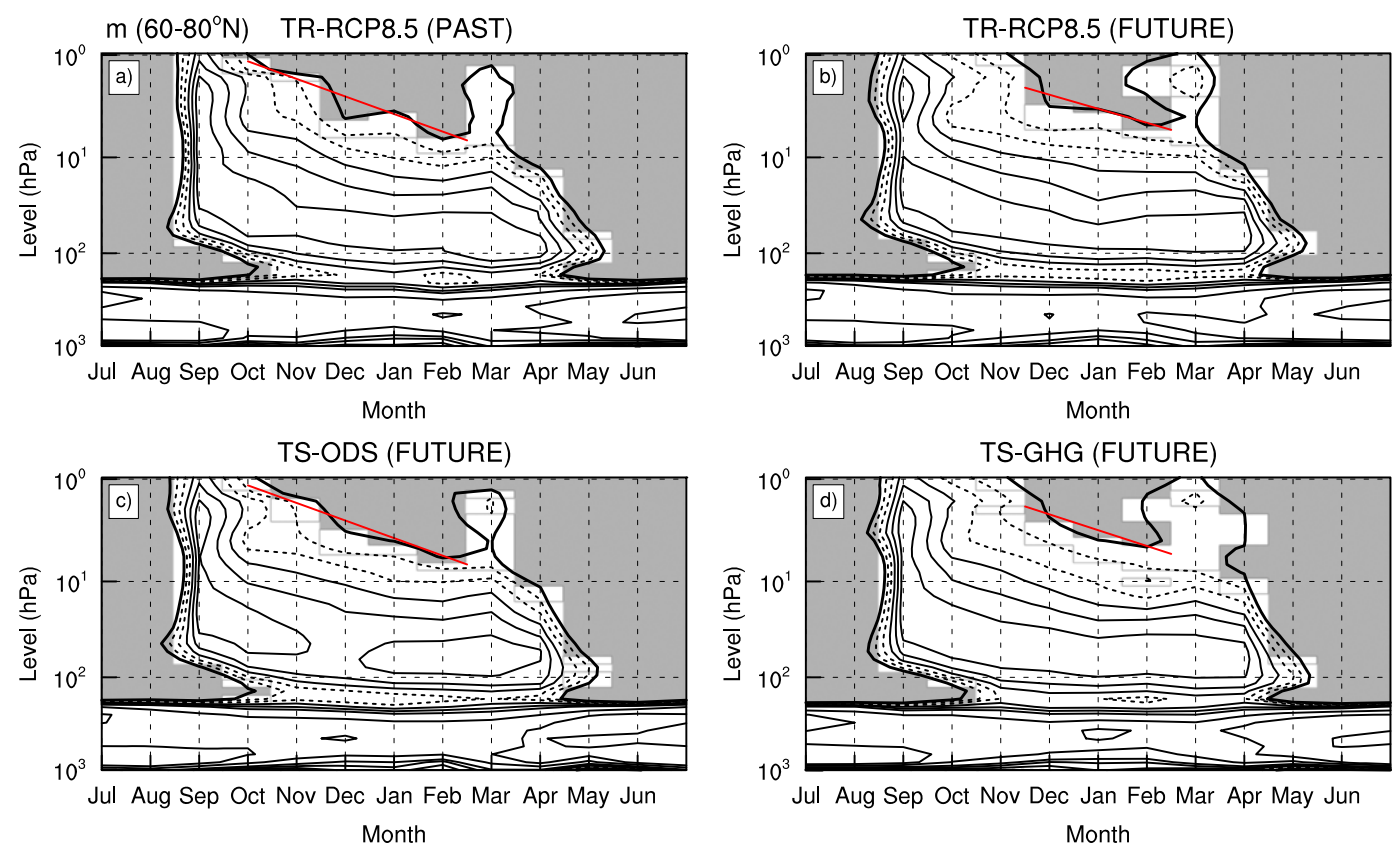

FIG. 4. The climatological vertical wavenumbers $m$ averaged between $60^{\circ}$ and $80^{\circ} \mathrm{N}$ for (a) TR-RCP8.5 past (1960-99), (b) TR-RCP8.5 future (2060-99), and two TS experiments with future (c) ODS and (d) GHG forcings. The vertical wavenumbers $\left(10^{-5} \mathrm{~m}^{-1}\right)$ are contoured with 0.01 (thick line), 2 and 4 (dashed lines), and 6-30 with intervals of 3 (thin lines). Finally, the shading indicates the regions of wave evanescence in vertical directions $(m<0)$. The red solid lines indicate the approximate linear descent rate of the vertical reflecting surface.

troposphere in the model. However, in the future, the downward wave events occur only over a shorter winter period from January to March, with no statistically significant signals in early winter (Fig. 3b). The overall wave coupling correlations in the future are lower compared to the recent past, indicating a significant reduction of downward wave activity from the stratosphere to the troposphere.

To examine whether the changes in the future timing of downward wave activity obtained from the transient simulation are attributed mainly to GHGs, we repeated the same diagnostics for two 40-yr TS experiments with different combinations in prescribed future surface emissions of the ODSs and GHGs. The TS simulations suggest that weaker downward wave signals in the future are mainly due to increases in GHG forcing (Figs. 3c,d). In particular, in the experiment with future ODS changes only (TS-ODS), downward wave signals were notably more persistent over a longer period (from December through April; Fig. 3c), with a pattern resembling the seasonal variation of downward wave signals in the recent past. In contrast, weak and less persistent downward wave signals were observed in the experiment with an increase in GHGs only (TS-GHG; Fig. 3d). We note that the high correlation from April to June for negative time lags in the TS-GHG experiment is not related to downward wave signals, but rather to a nonlinear wave reflection due to the vortex break up, since the vertical reflecting surface during this period (Fig. 4) is not bounded by the meridional waveguide (see Fig. S1d in the online supplemental material). The overall results suggest that a future decrease in the occurrence of downward wave activity in the $\mathrm{NH}$ is mainly attributed to increased GHG forcing alone, whereas ODS only plays a minor role.

To ensure that the downward-propagating wave signals found in Fig. 3 are associated with DWC events, we examine a month-to-month variation of the vertical reflecting surface and meridional waveguide. Note that the DWC occurs only when the vertical reflecting surface is bounded by a meridional waveguide in the lower stratosphere. Figure 4 shows the climatological vertical wavenumbers $m^{2}$ averaged from $60^{\circ}$ to $80^{\circ} \mathrm{N}$ for both the TR-RCP8.5 and TS simulations. In the past, the stratospheric reflecting surface persists from early to late winter (from October to March; Fig. 4a). This vertical reflecting surface is bounded by the extended meridional waveguide from November to March (Fig. S1a), allowing more favorable conditions for the occurrence of DWC during this period. By combining the period of bounded wave geometry and the wave coupling correlation (Figs. 3 and 4), the active period of DWC in the 
past is from November to March. The significant downward wave correlations in October and April are not associated with DWC, but are rather due to nonlinear wave dynamics (e.g., due to overreflection from a critical surface).

In the future, the vertical reflecting surfaces occur only from December to March (Fig. 4b), while the meridional waveguide exhibits the same seasonal evolution as in the past (Fig. S1b). This indicates that the favorable period for the DWC (based on the configuration of bounded wave geometry) is from December to March. By combining the period of bounded wave geometry and the wave coupling correlation (Figs. 3 and 4), we can conclude that the active period of DWC in the future is only from January to March. We further show that by using the TS simulations, the future changes in the reflecting surface are mainly attributed to GHG forcing (Fig. 4d), dominating the opposing influence of ozone recovery (Fig. 4c).

\section{d. Mechanisms for changes in the seasonality of DWC events}

The former analysis showed that there is a significant reduction of DWC events in the future, with a shift of their timing toward late winter (Figs. 3 and 4). To elucidate the mechanisms responsible for a decreased DWC activity in the future, we first analyze the trend in EP flux divergence, the vertical component of the EP flux $(\mathrm{Fz})$, and vertical wavenumbers in both the transient warming and control simulations. We then analyze the frequency of SSW and heat flux events in order to better understand the effect of wave absorption on the mean flow.

Although there is a clear reduction of the future DWC signal from early to midwinter (NDJ), the wave geometry shows a reflecting configuration [though the high latitude meridional waveguide is shallower during these months in the future (Fig. S1)]. This suggests that wave geometry changes cannot explain the reduction in the wave-coupling correlation in Fig. 3 in general, nor in particular for the early winter conditions. To further examine this, we analyze the trend in wave-1 EP-flux divergence $\mathrm{Fz}$ and vertical wavenumbers in NDJ as shown in Fig. 5. We do see that EP-flux wave-1 convergence is enhanced in the future (Fig. 5a). The increased wave convergence, in the first order, reflects increased wave absorption by the mean flow. Assuming there is no internal source of wave activity in the stratosphere, increased wave absorption simply results in a reduction in downward wave reflection by the mean flow and thus, decreased DWC events. In addition, the strengthening of wave absorption is accompanied by enhanced upward wave propagation from the troposphere into the stratosphere, as indicated by a positive

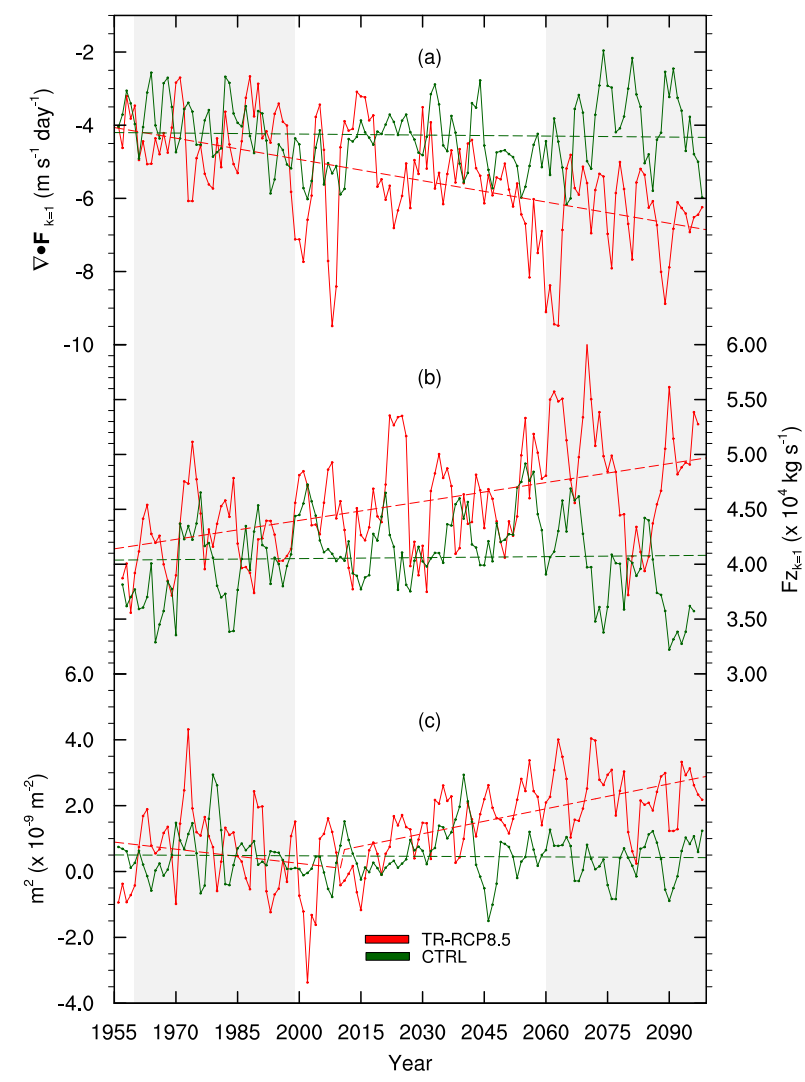

FIG. 5. NDJ mean of (a) wave-1 EP flux divergence averaged over $10-0.1 \mathrm{hPa}$ and $60^{\circ}-80^{\circ} \mathrm{N}$, (b) the vertical component of EP flux vectors at $100 \mathrm{hPa}$ averaged over $40^{\circ}-70^{\circ} \mathrm{N}$, and (c) vertical wavenumbers averaged over $5-1 \mathrm{hPa}$ and $60^{\circ}-80^{\circ} \mathrm{N}$ from the TRRCP8.5 (red) and CTRL (green) simulations. The straight dashed lines indicate linear best-fit regression (trend).

trend in Fz (see Fig. 5b), and by the positive trend in vertical wavenumber over the last decades, which altogether indicate a favorable condition for upward wave propagation in the future instead of downward reflection (Fig. 5c). This is again consistent with the wave coupling correlation in Figs. 3a and 3b, showing insignificant DWC events in early winter in the future. We also note that the future changes of $m^{2}$ in early winter are associated with changes in vertical shear of the zonalmean wind $U_{z}$ in the upper stratosphere. This is supported by a significant positive correlation between $m^{2}$, and $U_{z}$ and $\bar{q}_{y}$ (see Table 2). In contrast to transient warming simulation, we found no significant trends from the control simulation, suggesting that increased wave absorption in the stratosphere is induced mainly by future anthropogenic forcing. Our results so far suggest that the significant reduction of DWC in the future, in particular during early winter, can be associated with enhanced wave absorption in the stratosphere (Figs. 2 and 5), with stronger absorption concentrated in early winter. 
TABLE 2. Statistical features of the $60^{\circ}-80^{\circ} \mathrm{N}$ from November to December means of the 5-1-hPa mean $m^{2}$ and the 10-1-hPa means of zonal-mean wind shear $U_{z}$ and curvature $U_{z z}$, Brunt-Väisälä frequency $N^{2}$, and the meridional gradient of potential vorticity $\bar{q}_{y}$. Correlations significant at the $95 \%$ level based on a two-sided Student's $t$ test, assuming each year is independent, are in boldface.

\begin{tabular}{cccc}
\hline \hline Variables & Correlation with $\left\langle m^{2}\right\rangle$ & $|t|_{\text {val }}$ & Probability \\
\hline$\left\langle m^{2}\right\rangle$ & 1.000 & $\infty$ & 1.00 \\
$\left\langle U_{z}\right\rangle$ & $\mathbf{0 . 3 7 9}$ & 3.98 & 0.99 \\
$\left\langle U_{z z}\right\rangle$ & -0.185 & 1.05 & 0.53 \\
$\left\langle N^{2}\right\rangle$ & 0.004 & 0.05 & 0.39 \\
$\left\langle\bar{q}_{y}\right\rangle$ & $\mathbf{0 . 3 1 6}$ & 3.48 & 0.96 \\
\hline
\end{tabular}

Nevertheless, one can argue that the basic state itself is, in turn, altered by the waves and thus affects DWC. For example, increased wave absorption in the future can lead to enhanced SSW events and thus results in more downward wave reflection events. To investigate this possibility, we calculate the frequency of SSW events in the recent past and in the future from the TRRCP8.5 simulation and decompose these into reflective and absorptive types of SSW, following the definition of Kodera et al. (2016) (Figs. 6a-c). The reflective SSW is defined when the heat flux (zonal wavenumber 1 averaged over $45^{\circ}-75^{\circ} \mathrm{N}$ at $100 \mathrm{hPa}$ ) remains negative for more than 2 out of 7 days, on and after the maximum temperature during an SSW event, while the rest are classified as absorptive types. We found that there is a significant increase in SSW events in the future, compared to the past, where the frequency is dominated by absorptive SSW events (Figs. 6a-c). Thus, enhanced wave absorption in the future is mainly manifested by increased absorptive SSW events (rather than reflection), with more events concentrated in early winter. In addition, during absorptive SSW events, the vertical reflecting surface disappears or is located higher in the upper stratosphere, compared to reflective SSW events that are located in the lower stratosphere (not shown). Thus, a delay in the development of the midstratospheric reflecting surface in the future could be associated with stronger absorptive SSW events in early winter. Furthermore, we also calculate the frequency of upward-propagating wave events, which are defined by heat flux values (averaging over $45^{\circ}-75^{\circ} \mathrm{N}$ at $100 \mathrm{hPa}$ ), exceeding the 90th-percentile value of the daily distribution. The events are further decomposed into long (short) wave pulse events. The long (short) wave pulse events are defined when the positive heat flux persists for more (fewer) than 10 days after the central date. Harnik (2009) showed that long pulses of the upward wave activity could potentially cause warming events, while short pulses could lead to reflection. Our results show that there is a significant increase in upward wave activity, with long pulses in the future and with more (a) Major SSW

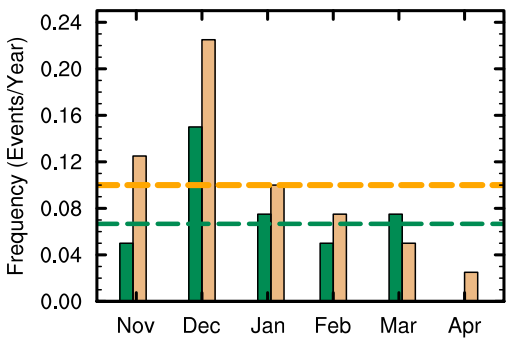

(d) $v^{\prime} T^{\prime}{ }_{100 \mathrm{hPa}}$

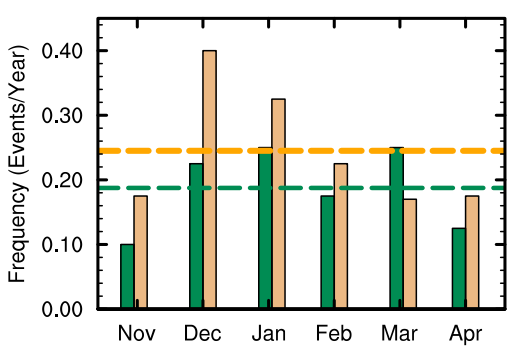

(b) Absorptive SSW

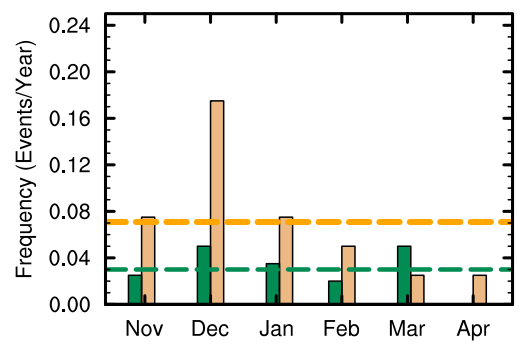

(e) $v^{\prime} T^{\prime}{ }_{100 \mathrm{hPa}}>10 \mathrm{~d}$ (long pulses)

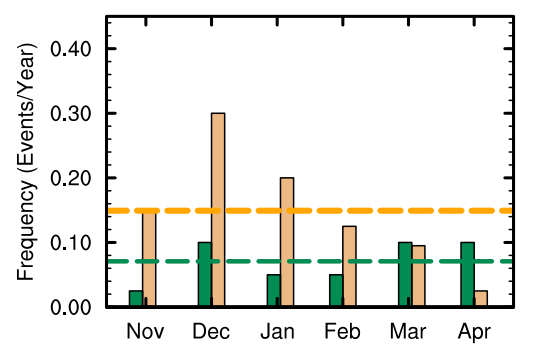

(c) Reflective SSW

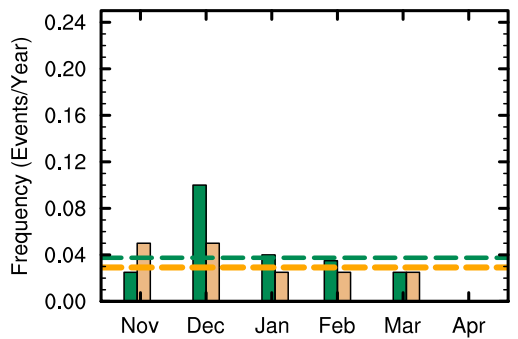

(f) $v^{\prime} T^{\prime}{ }_{100 \mathrm{hPa}}<10 \mathrm{~d}$ (short pulses)

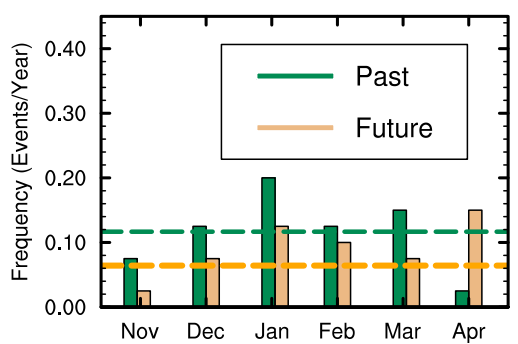

FIG. 6. The frequency of major warmings and upward heat flux events in NH winter months in the TR-RCP8.5 simulation for the past (1960-99, orange) and the future (2060-99, dark green). (a) Total frequency of major warming events and their decomposition into (b) absorptive and (c) reflective events. (d) The frequency of upward heat flux $\left(\overline{v^{\prime} T^{\prime}}>0\right)$ events at $100 \mathrm{hPa}$ and their decomposition into upward waves with (e) long and (f) short pulses. The horizontal dashed lines indicate the mean of the frequency. 
TABLE 3. Statistics of the daily distribution of wave- 1 heat flux averaged from $60^{\circ}$ to $90^{\circ} \mathrm{N}$ at $50 \mathrm{hPa}$ during JFM from the TR-RCP8.5 experiment for the past and future periods.

\begin{tabular}{lccccc}
\hline \hline Period & Mean & Std dev & 5th percentile & 95th percentile & Kolmogorov-Smirnov test $p$ value \\
\hline Past & 18.66 & 24.43 & -13.94 & 60.51 & 1.00 \\
Future & 20.41 & 25.11 & -11.23 & 67.50 & 0.04 \\
\hline
\end{tabular}

events concentrated in early winter, from November to January. These results are, therefore, consistent with enhanced wave absorption, increased absorptive SSWs, and reduced DWC events in the future, with more events concentrated in early winter.

In summary, our results show that a future decrease in DWC events could, in general, be associated with enhanced wave absorption in the stratosphere. The enhanced wave absorption leads to more absorbing SSW events, with more events concentrated in early winter. This early winter condition could lead to a delay in the development of the upper-stratospheric reflecting surface, resulting in a shift of the seasonal cycle of DWC toward late winter in the future.

\section{Tropospheric impact of DWC in the future}

Our previous results showed that DWC events are weaker in the future, with a shift of their timing toward late winter. Here, we examine whether the reduction of DWC events in the future has a potential impact on the tropospheric circulation and surface climate. We focus our analysis on the most active winter season, JFM, as it is a favorable period for planetary wave coupling in the NH (e.g., Perlwitz and Harnik 2003; Lubis et al. 2016a, 2017) and as a period where both the recent past and the future RCP8.5 experiments exhibit significant DWC signals in the troposphere, but weaker DWC activity in the future (see Figs. 3a,b).

\section{a. Impact on the tropospheric circulation}

Previous studies have shown that extreme negative wave- 1 heat flux in the stratosphere can be used to isolate the tropospheric impacts of DWC (e.g., DunnSigouin and Shaw 2015; Lubis et al. 2016a, 2017). In this study, the impact of individual DWC events on the tropospheric circulation is examined by looking at composites of various atmospheric and surface fields around the central events. The statistics of the highlatitude wave- 1 heat flux distribution for the RCP8.5 simulation for the past and future periods are listed in Table 3. The 5th- (95th) percentile values in Table 3 indicate the heat flux value below which $5 \%(95 \%)$ of each period's total heat flux distribution can be found. Consistent with our previous findings, there is significant decreased (increased) downward (upward) wave activity in the future, compared to the past. In particular, the wave- 1 heat flux magnitude at the 5 th percentile is lower by about $19.4 \%$, compared to the past, while the wave- 1 heat flux at the 95 th percentile is higher by $10.4 \%$, compared to the past.

Figure 7 shows the composites of $500-\mathrm{hPa}$ geopotential height (Figs. 7a,d), 500-hPa zonal-mean wind (Figs. 7b,e), and mean sea level pressure (MSLP) (Figs. $7 \mathrm{c}, \mathrm{f}$ ) anomalies north of $20^{\circ} \mathrm{N}$ during the time when DWC impact on the troposphere maximizes (from days -3 to 3 ). In the past, the spatial pattern of the $500-\mathrm{hPa}$ geopotential height and sea level pressure anomalies resembles a clear wave- 1 pattern with a node in the midlatitudes. In particular, over the North Atlantic sector, the signals project more onto the positive phase of the NAO-like pattern (rather than onto the negative phase), which is characterized by a seesaw shape (a dipole pattern) between mid- and high latitudes (Fig. 7a). This signature is further illustrated in the composite $500-\mathrm{hPa}$ zonal wind anomalies, which show a clear strengthening and poleward shift of the tropospheric jet over the North Atlantic basin (Fig. 7b). The corresponding sea level pressure anomalies exhibit a zonally asymmetric structure similar to that of the $500-\mathrm{hPa}$ geopotential height anomalies, being consistent with a quasi-barotropic, tropospheric NAO-like structure over the North Atlantic sector during the DWC events (Shaw and Perlwitz 2013). In addition, there are also significant signals in the North Pacific sector that reflect the potential impacts of wave reflection on the growth rate of baroclinic wave activity and the circulation over this region. The associated circulation change is characterized by an equatorward shift of the tropospheric jet. This result is consistent with the impact of DWC on tropospheric circulation obtained from reanalysis and model studies (e.g., Shaw and Perlwitz 2013; Shaw et al. 2014; Dunn-Sigouin and Shaw 2015).

In the future, the surface influence of DWC that resembles the tropospheric dipole-like pattern over the North Atlantic shifts eastward, relative to the patterns found in the past. In particular, the poleward shift of the tropospheric zonal-mean wind anomalies is located more to the east of the North Atlantic basin (Figs. 7e,h), which is consistent with the eastward shift of geopotential height anomalies at $500 \mathrm{hPa}$ (Figs. 7d,g). Likewise, the dipole pattern in the sea level pressure 

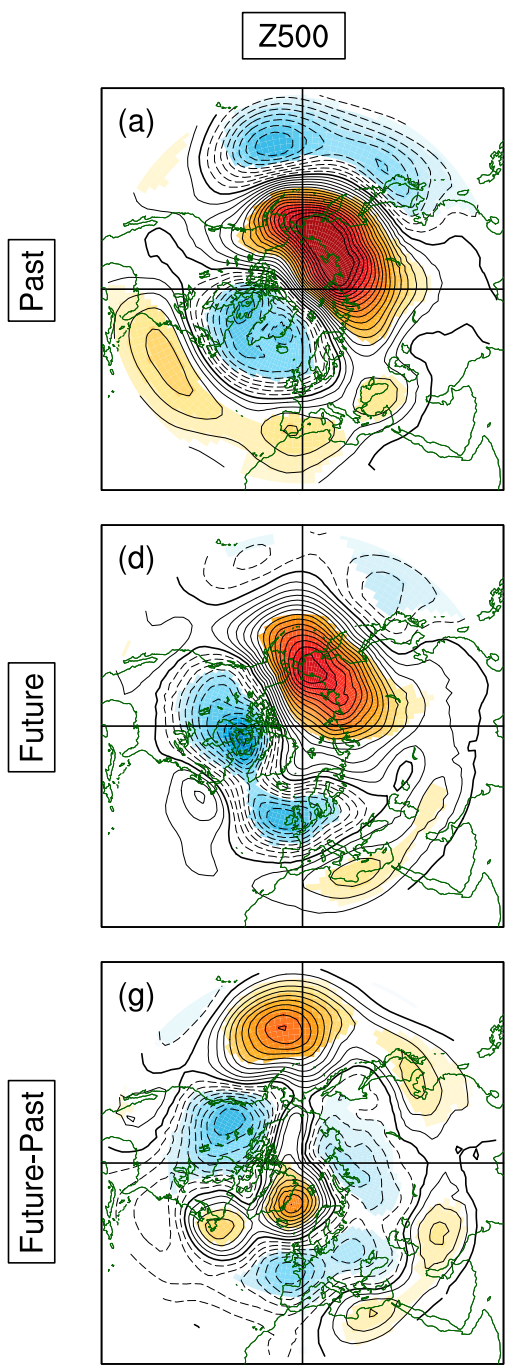

$[\mathrm{x} 10 \mathrm{~m}]$

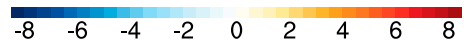

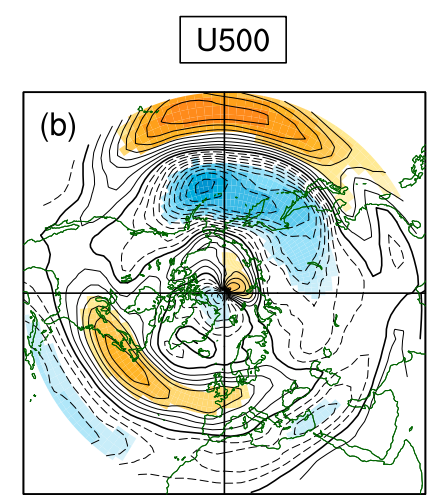
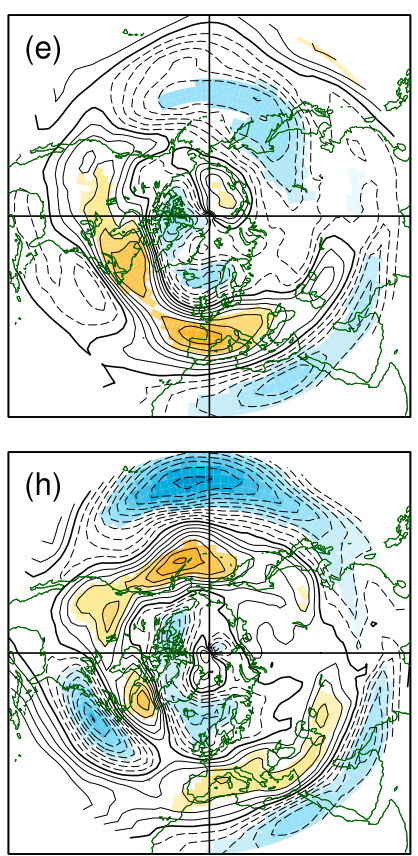

$[\mathrm{m} / \mathrm{s}]$

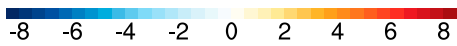

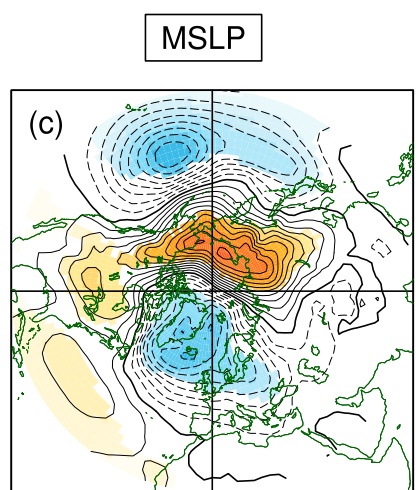
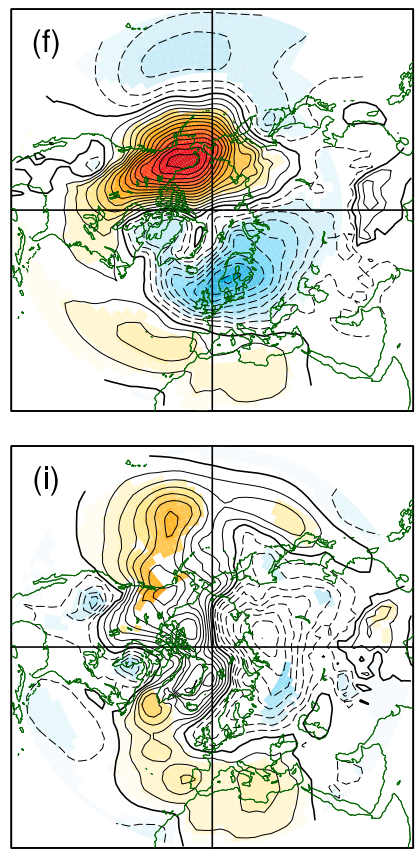

$[\mathrm{hPa}]$

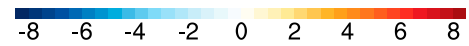

FIG. 7. The composites of (a),(d) 500-hPa geopotential height (Z500), (b),(e) 500-hPa zonal wind (U500), and (c),(f) MSLP anomalies during the period of maximum DWC impact on the troposphere (from days -3 to 3) in JFM for (top) TR-RCP8.5 past (1960-99) and (middle) TR-RCP8.5 future (2060-99). (g)-(i) The difference between the future and the past of the respective anomalies. Contour intervals are $10 \mathrm{~m}$ for $\mathrm{Z} 500,1 \mathrm{~m} \mathrm{~s}^{-1}$ for U500, and $1 \mathrm{hPa}$ for MSLP. The zero contour is omitted. The color shadings are only drawn for anomalies that are statistically significant at the $95 \%$ confidence level, according to a 1000 -trial Monte Carlo test.

anomalies also shifts eastward (Figs. 7f,i). In the North Pacific, the easterly wind anomalies weaken substantially and extend more to the south, compared to the past (Fig. 7h), suggesting a weakening of the westerlies on the equatorward flank of the jet in the future (see Fig. 9b later). These results are not sensitive to the DWC event definition or to the number of the events used for the composite calculation. In particular, if we randomly select the same number of composite members in the past as in the future, the differences in the spatial structures and magnitudes of the tropospheric responses to DWC remain the same. Qualitatively similar results are found using the DJF winter season (not shown).

Interestingly, one might expect that the tropospheric and surface responses to DWC over the North Atlantic sector in the future will be weaker as a result of decreased DWC events. However, we found that the anomalous strength of the tropospheric response to DWC over this region is relatively similar to that of the past (e.g., by comparing the strength of the westerly wind anomalies in the past and in the future over the North Atlantic region), but with the patterns shifted to 

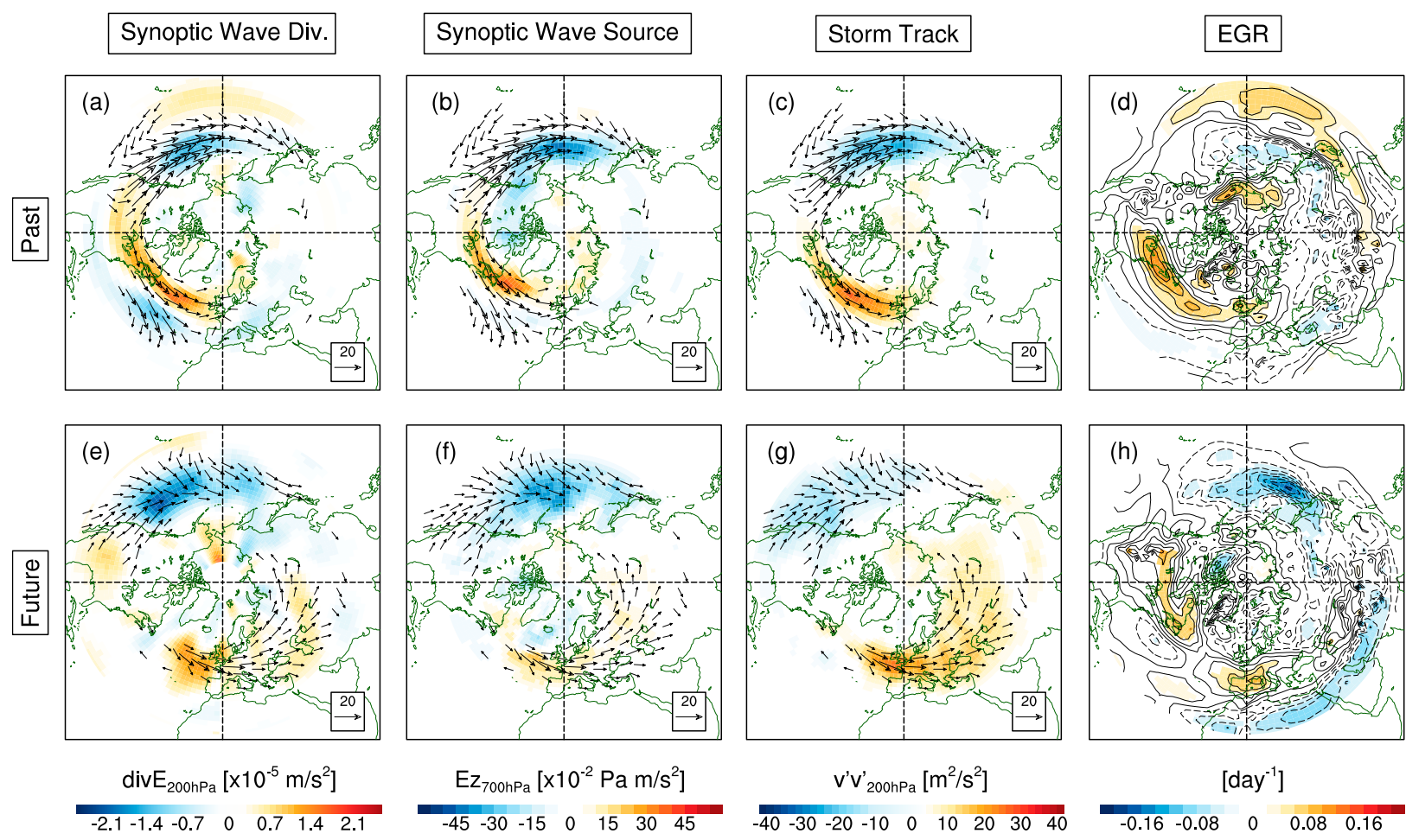

$\left[\right.$ day $\left.^{-1}\right]$

FIG. 8. The composites of the (a),(e) 200-hPa synoptic wave divergence, (b),(f) 775-hPa synoptic wave source, (c),(g) 200-hPa storm track, and (d),(h) 700-hPa EGR anomalies during the period of maximum DWC impact on the troposphere (from days -3 to 3 ) in JFM for (top) TR-RCP8.5 past (1960-99) and (bottom) TR-RCP8.5 future (2060-99). The vectors indicate the horizontal component of $\mathbf{E}$ vectors (Fx, Fy) at $200 \mathrm{hPa}$. The vertical component of $\mathbf{E}$ vectors in (b) and (f) is calculated by $-f \overline{v^{\prime} \theta^{\prime}}(\partial \theta / \partial p)^{-1}$ representing the synoptic wave source, where the positive (negative) values indicate upward (downward) synoptic wave fluxes. The color shading in (c) and (g) indicates the upper-level storm track anomalies $\overline{v^{\prime} v^{\prime}}$ at $200 \mathrm{hPa}$. The EGR maximum is calculated as $0.31|f||\partial u / \partial z| / N$. The shadings are only drawn for anomalies that are statistically significant at the $95 \%$ confidence level, according to a 1000-trial Monte Carlo test.

the east. In particular, the westerly anomaly center (over the North Atlantic sector) weakened significantly and shifted eastward into the Mediterranean. This suggests that other factors besides the frequency and strength of the downward wave propagation from the stratosphere to the troposphere influence the tropospheric response to DWC. A recent study by Lubis et al. (2016a) showed that internal tropospheric dynamics involving feedbacks from synoptic-scale eddy activity and atmosphereocean interaction were central to the responses, with the synoptic-scale eddy-driven accelerations being an order of magnitude larger than the directly induced planetary-scale-driven accelerations. We thus proceed to examine those feedbacks in the following section.

\section{b. Mechanisms of the tropospheric impact of DWC}

In this section, we aim to understand the dynamical mechanisms leading to the change in tropospheric DWC signal in the future. For this, we examine the contribution of 3D synoptic-scale (baroclinic) waves and 3D planetary-scale waves on the mean flow, similar to Lubis et al. (2016a).
Figure 8 shows the composites of the anomalous synoptic-scale divergence at $200 \mathrm{hPa}$, alongside the horizontal component of the $\mathbf{E}$ vectors (representing the influence of the synoptic-scale eddies on the horizontal large-scale flow; Figs. 8a,d), the anomalous vertical component of the $\mathbf{E}$ vectors at $775 \mathrm{hPa}$ (representing the source of synoptic-scale eddies; Figs. 8b,e), the anomalous synoptic meridional wind variance at $200 \mathrm{hPa}$ (representing the upper-level storm-track strength; Figs. 8c,f) and the synoptic Eady's growth rate (EGR) anomalies at $700 \mathrm{hPa}$ (representing the baroclinic instability in the troposphere; Figs. 8d,h) for the past (Figs. 8a-d) and future (Figs. 8e-h). In the past, we see that the synoptic eddy-induced accelerations, as shown by a divergence of $\mathbf{E}$ vectors, largely explain the poleward shift of the tropospheric wind anomalies over the North Atlantic sector (Figs. 8a, 7b). The magnitude of this acceleration is about 10 times larger than those due to planetary-scale waves (see Fig. S2a). Consistent with Lubis et al. (2016a), the anomalous acceleration pattern induced by synoptic-scale eddy anomalies (Fig. 8a) is accompanied by poleward shift of the tropospheric 
synoptic wave source (Fig. 8b) and the associated storm track anomalies (Fig. 8c). These mean flow baroclinicity anomalies are consistent with a poleward shift of the EGR anomalies, which are mainly driven by changes in the vertical wind shear induced by DWC (see Figs. S4a,b). In the North Pacific, the convergence of synopticscale waves (Fig. 8a) mostly explains the easterly wind anomalies in this region (Fig. 7b). This anomalous deceleration pattern induced by synoptic-scale waves, as shown by a convergence of $\mathbf{E}$ vectors, is accompanied by a poleward shift of the negative tropospheric synoptic wave source (Fig. 8b) and the associated storm track anomalies (Fig. 8c).

In the future, the location of the synoptic-scale divergence over the North Atlantic shifts to the east, compared to the patterns observed in the recent past (Figs. 7a,b). This is consistent with the shift of the tropospheric flow responses to DWC over the North Atlantic sector (Figs. 7d-f). In particular, the synoptic wave divergence anomalies (divergence of $\mathbf{E}$ vectors) explain the peak of zonal wind anomalies over western Europe (Fig. 7e) and the extended pattern into eastern Europe. The magnitude of the synoptic eddy divergence is much larger than the accelerations by planetary-scale waves (see Fig. S2b), suggesting that synoptic-scale eddies play a more important role in setting the tropospheric response to DWC in the future [consistent with the mechanism proposed by Lubis et al. (2016a)]. Furthermore, we also found that the eastward shift of the synoptic-scale divergence over the North Atlantic sector is consistent with the shift of the tropospheric synoptic wave source (Fig. 8f), the storm track anomalies (Fig. 8g), and the lower-level baroclinicity (Fig. 8h) to the east. The lower-level baroclinicity anomalies in the future are attributed to both vertical wind shear and static stability, in contrast to the past, which is driven mainly by vertical wind shear (see Figs. S3c,d). These results suggest that the tropospheric response to DWC over the North Atlantic sector in the future is associated with the eastward shift of the baroclinic eddy-mean flow interaction in response to anthropogenic climate change. In the North Pacific, the southward extension of easterly wind anomalies during DWC is consistent with the extension of the synoptic-scale wave convergences to the south (Fig. 8e). This anomalous deceleration is also consistent with the weakening of synoptic-scale wave activity and the storm track over the North Pacific in the future (Figs. 8f,g). The weakening of baroclinic wave activity is also consistent with decreased EGR in the western boundary of the North Pacific basin (Fig. 8h).

The results so far show that the tropospheric response to DWC events has a very different spatial pattern in the future, and this change in pattern is similar for the mean flow quantities (zonal wind, surface pressure, and geopotential height) and for the synoptic eddies and their fluxes. This suggests that the tropospheric response to DWC is associated with a change in synoptic-scale eddy feedbacks. However, it is not clear why the pattern of the synoptic-scale eddy feedback differs compared to the past (i.e., shifting more to the east). Therefore, it is worth checking if changes in DWC-induced synoptic-scale eddymean flow interaction are adjusted by the changes in the mean states (both the mean flow and storm track) in response to future anthropogenic climate change.

To answer this question, we analyzed the differences in the JFM mean zonal wind $\bar{u}$ and storm track $\overline{v^{\prime} v^{\prime}}$ at $200 \mathrm{hPa}$ between the future and the past (Fig. 9). In the North Atlantic, we can see that there is a poleward shift and an eastward extension of $\bar{u}_{200}$ and $\overline{v^{\prime} \boldsymbol{v}^{\prime}}{ }_{200}$, alongside the associated $\mathbf{E}$ vectors in the future (Figs. $8 \mathrm{a}-\mathrm{c}$ ). The eastward extension of the mid-high-latitude Atlantic eddy-driven jet toward western Europe is evident, with peaks of $\bar{u}_{200}, \overline{v^{\prime} v^{\prime}} 200$, and $\mathbf{E}$ vectors clearly shifting eastward, compared to the climatology from the past (Figs. 9d-f). Similar patterns as shown in the responses (Figs. 8c,f) can be confirmed by a long-term linear trend for each quantity (see Fig. S4), where the trends in the North Atlantic tropospheric jet and the storm track altogether shift poleward and extend eastward. In the North Pacific sector, the poleward shift in the storm tracks and the tropospheric jet are also consistent with the DWC's response being confined to mid-high latitudes and with no subtropical extension in the Pacific in the future, whereas in the past, there was a subtropical signal. These results suggest that the shift in the pattern of the DWC-induced synoptic-scale eddy-mean flow interaction in the midhigh-latitude troposphere in the future is adjusted by the inherent changes in the mean states (both mean flow and storm track) in response to anthropogenic climate change.

The eastward extension of the North Atlantic storm track in the future in our model can also be related to changes in the lower-level baroclinicity induced by local SST gradients, resulting in enhancing baroclinic wave activity and the associated impact on the mean flow. To test this hypothesis, we analyzed the differences in the SST gradient and Eady's growth rate between the future and past during winter (JFM) (Fig. 10). In Fig. 10, we can see that there is a weakening (strengthening) of the SST gradient in the southern (middle) part of the western Atlantic Gulf Stream front (Figs. 10a,b), which is consistent with the reduced (enhanced) EGR (Figs. 10c,d) and $\mathbf{E}$ vectors there (Figs. 9b,d). On the other hand, there is a strengthening of SST gradients to the east (around the North Sea), followed by enhanced EGR, suggesting an increased synoptic (baroclinic) wave generation over the North Sea and northwestern Europe (Figs. 9b,d). The strengthened baroclinicity over these 
(a) Past: $\mathrm{u}_{200 \mathrm{hPa}}$

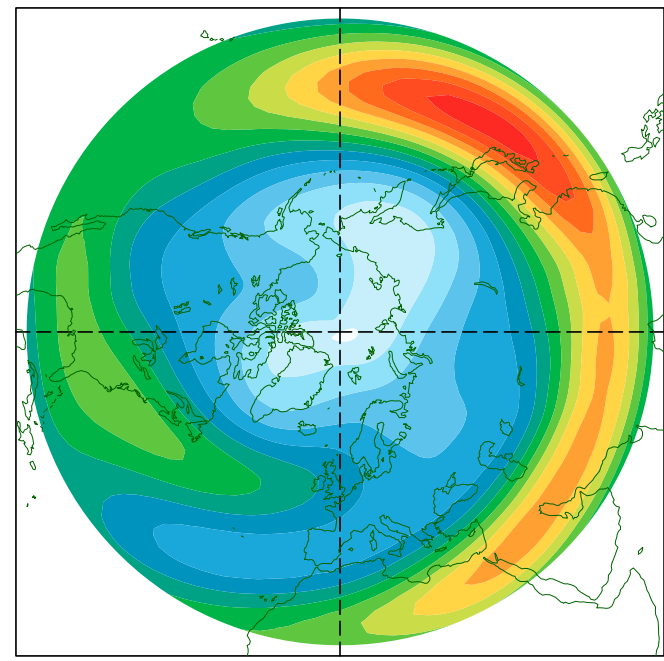

$[\mathrm{m} / \mathrm{s}]$

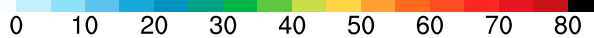

(c) Past: v'v' ${ }_{200 \mathrm{hPa}}$

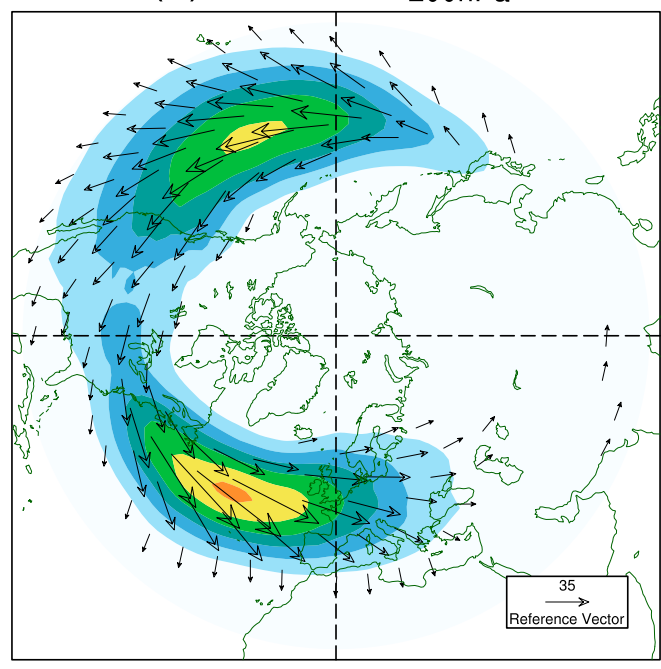

$\left[\mathrm{m}^{2} / \mathrm{s}^{2}\right]$

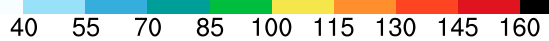

(b) Future - Past: $\mathrm{u}_{200 \mathrm{hPa}}$

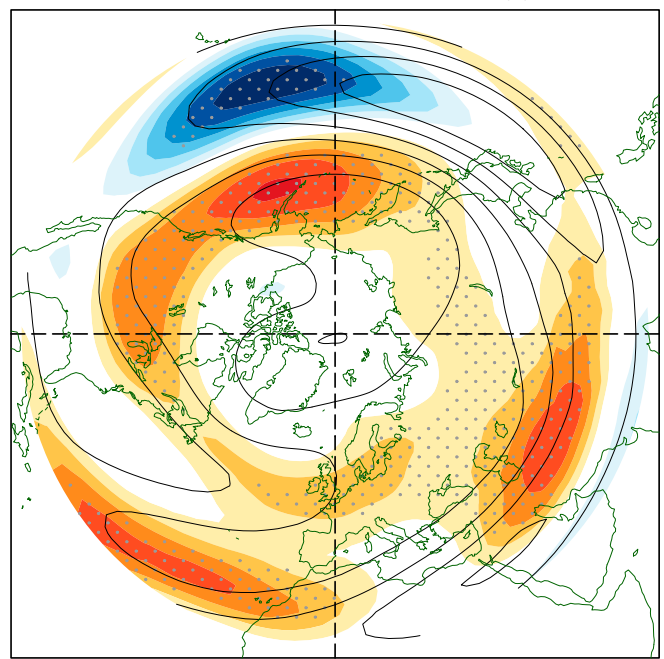

$[\mathrm{m} / \mathrm{s}]$

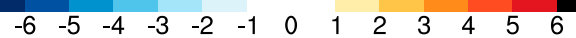

(d) Future - Past: v'v' $200 \mathrm{hPa}$

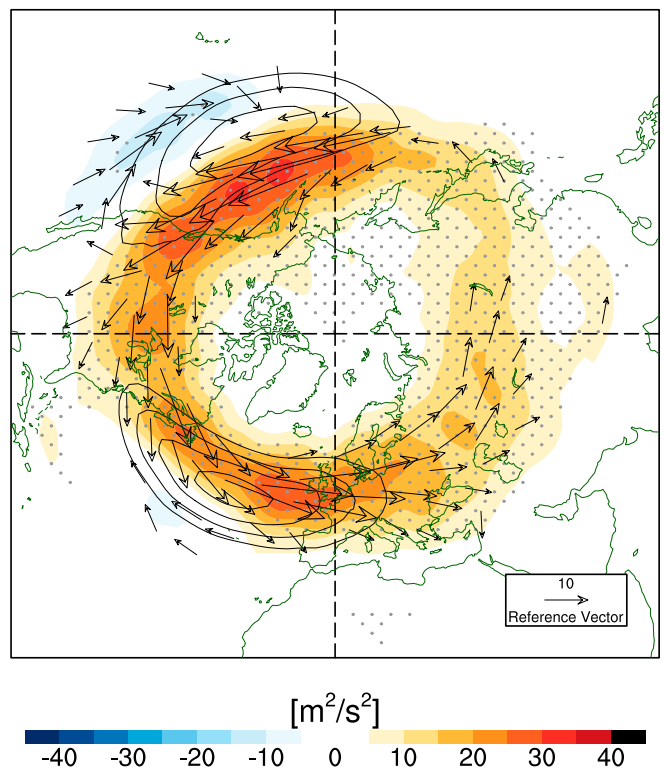

FIG. 9. The JFM mean 200-hPa zonal wind and 200-hPa storm track $\overline{\boldsymbol{v}^{\prime} \boldsymbol{v}^{\prime}}$ from (a),(c) the past and (b),(d) the response (future - past) from TR-RCP8.5. The black contour lines in (b) and (d) indicate a climatology from the past. The gray dots indicate the regions where the changes are significant at the $95 \%$ confidence level, according to a two-tailed $t$ test.

regions is consistent with the increased storm track and zonal wind (Figs. 9b,d). This hints that the eastward extension of the North Atlantic jet under future climate change could be also related to the shift of the lowerlevel baroclinicity and the associated synoptic-scale eddy-mean flow interaction.

\section{Summary and discussion}

This study examined the impact of future anthropogenic climate changes on DWC events in $\mathrm{NH}$ winter, particularly how their seasonality will change in the future and how different anthropogenic forcings (GHG 
(a) Past: $-\mathrm{SST}_{\mathrm{y}}$

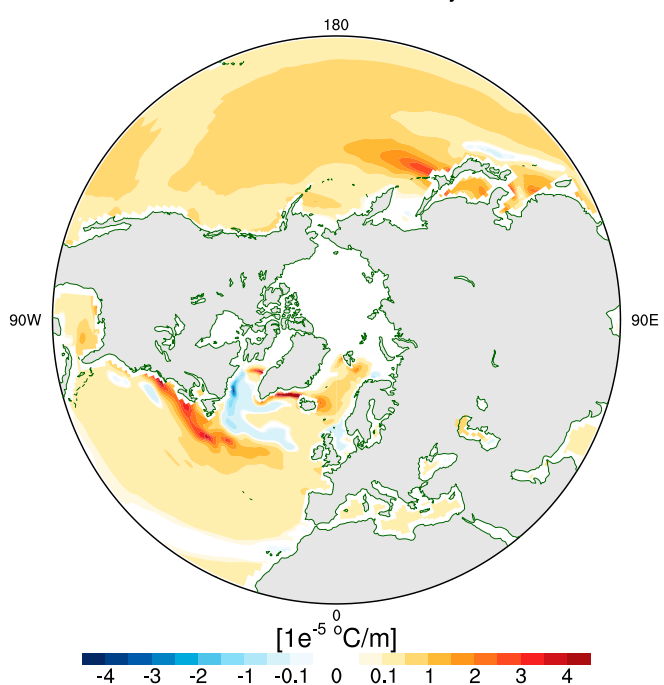

(c) Past: EGR

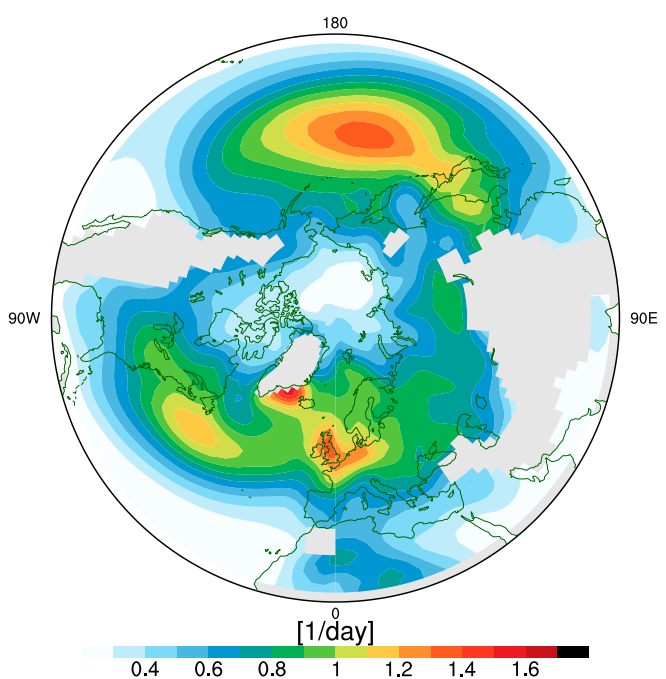

(b) Future - Past: -SST

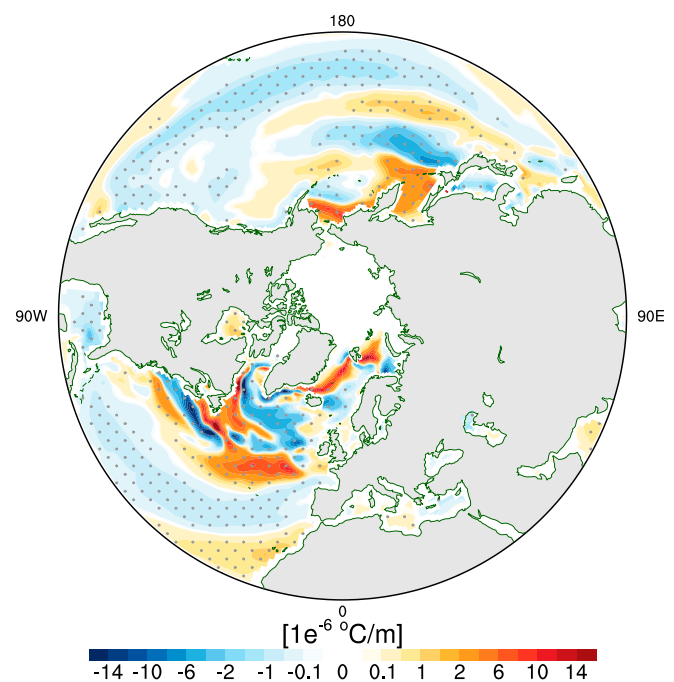

(d) Future-Past: EGR

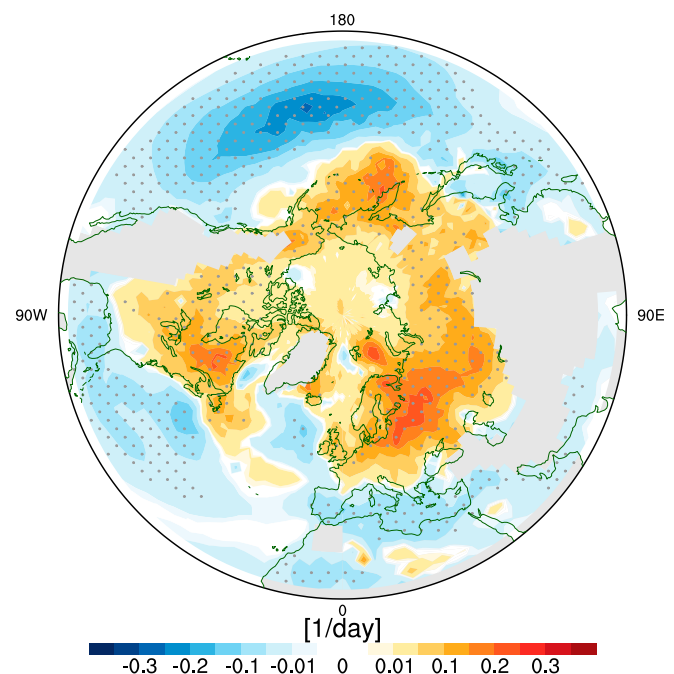

FIG. 10. The JFM mean meridional gradient of SST (SSTy) and EGR maximum at $925 \mathrm{hPa}$ from (a),(c) the past and (b),(d) the response (future - past) in TR-RCP8.5. The gray shaded regions indicate where the land or the "underground" grid points (i.e., $z>1 \mathrm{~km}$ ) have been excluded from the analysis. The SSTy value has been multiplied by -1 for a better comparison with the EGR's sign. The gray dots indicate the regions where the changes are significant at the $95 \%$ confidence level, according to a two-tailed $t$ test.

and ODSs) individually influence the occurrence of these events. Two long-term (145 years) fully coupled chemistryclimate model simulations with CESM1(WACCM) with fixed and time-varying anthropogenic forcings following the RCP8.5 scenario have been used to examine the impact of anthropogenic forcing on DWC. In addition, two TS experiments with a combination of past and future GHG or ODS concentrations were also used to isolate the influence of each anthropogenic forcing factor on DWC. In our analysis, the attribution of anthropogenic forcings on DWC was analyzed by examining the differences in background wind, wave-mean flow interaction, and a time-lagged vertical wave-1 coupling, as well as the evolution of wave geometry. Furthermore, the tropospheric impact of DWC in midwinter was investigated using a metric based on the stratospheric heat flux extremes. Summary points from our analysis are as follows:

- There is a significant change in the vortex mean state over the twenty-first century, characterized by a 
weaker and more disturbed polar vortex, with most changes occurring in early winter (Fig. 1). This is consistent with a significant increase in the EP flux convergence during that period (Fig. 2).

- There is statistically significant change in DWC frequency and its seasonality over the twenty-first century, when compared to the recent past. In the past, DWC occurs throughout the winter, with most events concentrated in DJF, but as GHG concentrations increase, DWC becomes significantly weaker, with more events concentrated in late winter, from February to March (Figs. 3a,b). Changes in GHG alone, without ODSs, can account for these changes (Figs. 3c,d and 4).

- The future decrease in DWC events by the end of the twenty-first century could, in general, be associated with enhanced wave absorption in the stratosphere (Figs. 2, 5, and 6). The enhanced wave absorption manifests as more absorbing SSW events, with more events concentrated in early winter (Fig. 6). This early winter condition could lead to a delay in the development of the upper-stratospheric reflecting surface during that period (Fig. 5), resulting in a shift in the seasonal cycle of the DWC toward late winter in the future.

- While the natural forcing factors, such as SST variability and the QBO, induce a change in the strength of the tropospheric response to DWC mostly over the North Atlantic (Lubis et al. 2016a), the increase in anthropogenic forcing (mainly due to GHG increases) changes the tropospheric response to DWC itself, with a large change in both ocean basins and a zonal shifting of the Atlantic center of action. This change in pattern is consistent with the trends in the climatology of the tropospheric jet and storm tracks, manifested as a shift in the main centers of eddy-mean flow interaction that shape the tropospheric response to DWC (Figs. 7-10).

A recent study by Lubis et al. (2016a) showed that the tropospheric response to DWC is dominated by eddymean flow feedbacks, which are excited by the initial downward wave reflection in the stratosphere. Thus, it is expected that an eastward shift of the storm track and jets will result in an eastward shift of the eddy feedbacks and is consistent with the tropospheric response to DWC in the future. It is also well established that the DWC induces strong positive NAO events (e.g., Lubis et al. 2016a; Shaw et al. 2014; Dunn-Sigouin and Shaw 2015) so that a reduction in downward reflection means a reduction in this source of positive NAO events. Our results, however, showed that while there is a significant reduction in DWC in the future, the strength of the NAO-like pattern does not significantly change; rather, it induces an eastward extension of the positive
NAO-like pattern. This suggests that other dynamical adjustments (outside of DWC) to global warming can be also important to determine the strength and dynamics of the NAO in the future.

We have yet to explain the mechanism that is responsible for the enhanced upward-propagating planetary waves in our warming simulation. Previous studies have shown that changes in the location of critical layers within the subtropical lower stratosphere cause an increase in upward-propagating planetary waves from the troposphere into the stratosphere (Shepherd and McLandress 2011). Such changes in planetary and synoptic wave breaking in the location of critical layers are mainly driven by tropical SST forcing (Oberländer et al. 2013; Ayarzagüena et al. 2013). It is also argued that future increases in tropical SSTs can enhanced upward planetary wave activity into the stratosphere through a positive interference of wave activity due to a deepening of the Aleutian low (Ayarzagüena et al. 2013). Thus, it is possible that the increased upward wave activity with long pulses, which causes an increase in wave absorption in the future, may be related to one of these processes. Further studies are required to check this possibility, and we leave this open for further investigation.

The results of the analysis also show that the North Atlantic storm track shifts poleward and extends farther east under future climate change, consistent with recent ocean-atmosphere coupled GCM studies (e.g., Woollings et al. 2012; Ciasto et al. 2016). Our model results suggest that the cause is likely due to the projected changes in local North Atlantic SST, resulting in the intensification and extension of the eddy-driven jet toward western Europe. A recent study by Ciasto et al. (2016) found that such a shift can be also due to the remote local SST changes, originating from the tropical western Pacific Ocean via Rossby wave trains. However, a clear attribution of that causality is difficult in our results because the analysis is performed on a fully coupled simulation. Therefore, further studies are required in order to better understand the origin of future changes in tropospheric jet shift in response to DWC (i.e., local vs remote influence): for example, by performing a comprehensive set of sensitivity experiments with a separate climate forcing, such as tropical or subtropical SST forcing only or sea ice forcing only.

This work can be viewed as a complementary study to that of Lubis et al. (2016a), who specifically examined the impact of the natural forcing factors, including SST and the QBO, on DWC and the associated surface impact in $\mathrm{NH}$ winter. In this study, we stressed that anthropogenic forcing factors indeed play important roles in controlling DWC and the associated surface climate 
in the NH. Previous studies also showed that the 11-yr solar cycle may play a role in perturbing the stratospheric mean state and the formation of the reflecting surface in the upper stratosphere (Matthes et al. 2006; Lu et al. 2017a,b). Therefore, understanding the role of solar forcing for the tropospheric impact of DWC is important and a subject of future investigation. A better understanding of the dynamical processes by which the stratosphere can influence the troposphere via planetary wave reflection has the potential to improve seasonal forecasting and climate prediction, thus leading to significant societal impacts.

Acknowledgments. We thank three anonymous reviewers for useful comments. We acknowledge support received from the German-Israeli Foundation for Scientific Research and Development under Grant GIF1151-83.8/2011. This work has also been partially performed within the Helmholtz University Young Investigators Group NATHAN funded by the Helmholtz Association through the President's Initiative and Networking Fund and the GEOMAR Helmholtz Centre for Ocean Research Kiel. Part of the work was done while $\mathrm{NH}$ was on sabbatical at Stockholm University, supported by a Rossby Visiting Fellowship from the International Meteorological Institute (IMI) of Stockholm University, Sweden. We would also like to thank Ted Shepherd and Edwin Gerber for useful discussions on the results during their visit at the GEOMAR, Kiel. The model simulations were performed at the German Climate Computing Centre [Deutsches Klimarechenzentrum (DKRZ)], Hamburg, and the NEC-HPC Linux Cluster at Christian-Albrechts Universität zu Kiel, Kiel.

\section{APPENDIX}

\section{Stationary Planetary Wave Forcing}

To quantify the drag exerted by stationary planetaryscale waves on the zonal-mean flow, the $3 \mathrm{D}$ wave activity flux (Plumb 1985) to diagnose the potential regional sources (sinks) and propagation characteristics of stationary planetary-scale wave activity is computed as follows:

$$
\mathbf{F}_{s}=\frac{p \cos \phi}{p_{o}} \times\left\{\begin{array}{l}
\frac{1}{2 a^{2} \cos ^{2} \phi}\left[\left(\frac{\partial \psi^{\prime}}{\partial \lambda}\right)^{2}-\psi^{\prime} \frac{\partial^{2} \psi^{\prime}}{\partial \lambda^{2}}\right] \\
\frac{1}{2 a^{2} \cos \phi}\left(\frac{\partial \psi^{\prime}}{\partial \lambda} \frac{\partial \psi^{\prime}}{\partial \phi}-\psi^{\prime} \frac{\partial^{2} \psi^{\prime}}{\partial \lambda \partial \phi}\right) \\
\frac{2 \Omega^{2} \sin ^{2} \phi}{N^{2} a \cos \phi}\left(\frac{\partial \psi^{\prime}}{\partial \lambda} \frac{\partial \psi^{\prime}}{\partial z}-\psi^{\prime} \frac{\partial^{2} \psi^{\prime}}{\partial \lambda \partial z}\right)
\end{array}\right\},
$$

where $\psi, \lambda, \phi, \Omega$, and $\theta$ are the streamfunction, longitude, latitude, Earth's rotation rate, and potential temperature, respectively, $p$ is pressure level, and $p_{o}$ is $1000 \mathrm{hPa}$. The overbar and prime with the $\mathbf{F}_{s}$ vectors denote the zonal mean and departure from it, respectively. The $\mathbf{F}_{s}$ vectors are parallel to the wave energy propagation direction, and its zonal mean is equivalent to the Eliassen-Palm flux (Plumb 1985). The 3D Plumb flux is calculated only for zonal wave components 1 and 2 .

\section{REFERENCES}

Ayarzagüena, B., U. Langematz, S. Meul, S. Oberländer, J. Abalichin, and A. Kubin, 2013: The role of climate change and ozone recovery for the future timing of major stratospheric warmings. Geophys. Res. Lett., 40, 2460-2465, https:// doi.org/10.1002/grl.50477.

Baldwin, M. P., and T. J. Dunkerton, 2001: Stratospheric harbingers of anomalous weather regimes. Science, 294, 581-584, https://doi.org/10.1126/science.1063315.

Bell, C. J., L. J. Gray, and J. Kettleborough, 2010: Changes in Northern Hemisphere stratospheric variability under increased $\mathrm{CO}_{2}$ concentrations. Quart. J. Roy. Meteor. Soc., 136, 1181-1190, https://doi.org/10.1002/qj.633.

Blackmon, M. L., 1976: A climatological spectral study of the $500 \mathrm{mb}$ geopotential height of the Northern Hemisphere. J. Atmos. Sci., 33, 1607-1623, https://doi.org/10.1175/ 1520-0469(1976)033<1607:ACSSOT>2.0.CO;2.

Bretherton, C. S., C. Smith, and J. M. Wallace, 1992: An intercomparison of methods for finding coupled patterns in climate data. J. Climate, 5, 541-560, https://doi.org/10.1175/ 1520-0442(1992)005<0541:AIOMFF $>2.0 . \mathrm{CO} ; 2$.

Butchart, N., J. Austin, J. R. Knight, A. A. Scaife, and M. L. Gallani, 2000: The response of the stratospheric climate to projected changes in the concentrations of well-mixed greenhouse gases from 1992 to 2051. J. Climate, 13, 21422159, https://doi.org/10.1175/1520-0442(2000)013<2142: TROTSC $>2.0 . \mathrm{CO} ; 2$.

Charlton-Perez, A. J., L. M. Polvani, J. Austin, and F. Li, 2008: The frequency and dynamics of stratospheric sudden warmings in the 21st century. J. Geophys. Res., 113, D16116, https://doi.org/ 10.1029/2007JD009571.

Charney, J. G., and P. G. Drazin, 1961: Propagation of planetaryscale disturbances from the lower into the upper atmosphere. J. Geophys. Res., 66, 83-109, https://doi.org/10.1029/ JZ066i001p00083.

Ciasto, L. M., C. Li, J. J. Wettstein, and N. G. Kvamstø, 2016: North Atlantic storm-track sensitivity to projected sea surface temperature: Local versus remote influences. J. Climate, 29, 6973 6991, https://doi.org/10.1175/JCLI-D-15-0860.1.

Dunn-Sigouin, E. and T. A. Shaw, 2015: Comparing and contrasting extreme stratospheric events, including their coupling to the tropospheric circulation. J. Geophys. Res. Atmos., 120, 1374-1390, https://doi.org/10.1002/2014JD022116.

Eichelberger, S. J. and D. L. Hartmann, 2005: Changes in the strength of the Brewer-Dobson circulation in a simple AGCM. Geophys. Res. Lett., 32, L15807, https://doi.org/ 10.1029/2005GL022924.

Gent, P. R., and Coauthors, 2011: The Community Climate System Model version 4. J. Climate, 24, 4973-4991, https://doi.org/ 10.1175/2011JCLI4083.1. 
Hansen, F., K. Matthes, C. Petrick, and W. Wang, 2014: The influence of natural and anthropogenic factors on major stratospheric sudden warmings. J. Geophys. Res. Atmos., 119, 8117-8136, https://doi.org/10.1002/2013JD021397.

Harnik, N., 2009: Observed stratospheric downward reflection and its relation to upward pulses of wave activity. J. Geophys. Res., 114, D08120, https://doi.org/10.1029/2008JD010493.

, and R. S. Lindzen, 2001: The effect of reflecting surfaces on the vertical structure and variability of stratospheric planetary waves. J. Atmos. Sci., 58, 2872-2894, https://doi.org/10.1175/ 1520-0469(2001)058<2872:TEORSO > 2.0.CO;2.

Hoskins, B. J., I. N. James, and G. H. White, 1983: The shape, propagation and mean-flow interaction of large-scale weather systems. J. Atmos. Sci., 40, 1595-1612, https://doi.org/10.1175/ 1520-0469(1983)040<1595:TSPAMF >2.0.CO;2.

Hurrell, J. W., and Coauthors, 2013: The Community Earth System Model: A framework for collaborative research. Bull. Amer. Meteor. Soc., 94, 1339-1360, https://doi.org/ 10.1175/BAMS-D-12-00121.1.

Kinnison, D. E., and Coauthors, 2007: Sensitivity of chemical tracers to meteorological parameters in the MOZART-3 chemical transport model. J. Geophys. Res., 112, D20302, https://doi.org/10.1029/2006JD007879.

Kodera, K., K. Yamazaki, M. Chiba, and K. Shibata, 1990: Downward propagation of upper stratospheric mean zonal wind perturbation to the troposphere. Geophys. Res. Lett., 17, 1263-1266, https://doi.org/10.1029/GL017i009p01263.

- H. Mukougawa, P. Maury, M. Ueda, and C. Claud, 2016: Absorbing and reflecting sudden stratospheric warming events and their relationship with tropospheric circulation. J. Geophys. Res. Atmos., 121, 80-94, https://doi.org/10.1002/ 2015JD023359.

Lean, J., G. Rottman, J. Harder, and G. Kopp, 2005: SORCE contributions to new understanding of global change and solar variability. The Solar Radiation and Climate Experiment (SORCE), G. Rottman, T. Woods, and V. George, Eds., Springer, 27-53.

Lu, H., L. J. Gray, I. P. White, and T. J. Bracegirdle, 2017a: Stratospheric response to the 11-yr solar cycle: Breaking planetary waves, internal reflection, and resonance. J. Climate, 30, 7169-7190, https://doi.org/10.1175/JCLI-D-17-0023.1.

— A. A. Scaife, G. J. Marshall, J. Turner, and L. J. Gray, 2017b: Downward wave reflection as a mechanism for the stratosphere-troposphere response to the 11-yr solar cycle. J. Climate, 30, 2395-2414, https://doi.org/10.1175/ JCLI-D-16-0400.1.

Lubis, S. W., K. Matthes, N.-E. Omrani, N. Harnik, and S. Wahl, 2016a: Influence of the quasi-biennial oscillation and sea surface temperature variability on downward wave coupling in the Northern Hemisphere. J. Atmos. Sci., 73, 1943-1965, https://doi.org/10.1175/JAS-D-15-0072.1.

- N.-E. Omrani, K. Matthes, and S. Wahl, 2016b: Impact of the Antarctic ozone hole on the vertical coupling of the stratosphere-mesosphere-lower thermosphere system. J. Atmos. Sci., 73, 2509-2528, https://doi.org/10.1175/ JAS-D-15-0189.1.

—, V. Silverman, K. Matthes, N. Harnik, N.-E. Omrani, and S. Wahl, 2017: How does downward planetary wave coupling affect polar stratospheric ozone in the Arctic winter stratosphere? Atmos. Chem. Phys., 17, 2437-2458, https://doi.org/ 10.5194/acp-17-2437-2017.

, C. S. Huang, N. Nakamura, N. Omrani, and M. Jucker, 2018: Role of finite-amplitude Rossby waves and nonconservative processes in downward migration of extratropical flow anomalies. J. Atmos. Sci., https://doi.org/10.1175/JAS-D-170376.1, in press.

Manzini, E., and Coauthors, 2014: Northern winter climate change: Assessment of uncertainty in CMIP5 projections related to stratosphere-troposphere coupling. J. Geophys. Res. Atmos., 119, 7979-7998, https://doi.org/10.1002/2013JD021403.

Marsh, D. R., M. J. Mills, D. E. Kinnison, J.-F. Lamarque, N. Calvo, and L. M. Polvani, 2013: Climate change from 1850 to 2005 simulated in CESM1(WACCM). J. Climate, 26, 73727391, https://doi.org/10.1175/JCLI-D-12-00558.1.

Matsuno, T., 1970: Vertical propagation of stationary planetary waves in the winter Northern Hemisphere. J. Atmos. Sci., 27, 871-883, https://doi.org/10.1175/1520-0469(1970)027<0871: VPOSPW $>2.0 . \mathrm{CO} ; 2$.

Matthes, K., Y. Kuroda, K. Kodera, and U. Langematz, 2006: Transfer of the solar signal from the stratosphere to the troposphere: Northern winter. J. Geophys. Res., 111, D06108, https://doi.org/10.1029/2005JD006283.

—, D. R. Marsh, R. R. Garcia, D. E. Kinnison, F. Sassi, and S. Walters, 2010: Role of the QBO in modulating the influence of the 11 year solar cycle on the atmosphere using constant forcings. J. Geophys. Res., 115, D18110, https://doi.org/ 10.1029/2009JD013020.

Meinshausen, M., and Coauthors, 2011: The RCP greenhouse gas concentrations and their extensions from 1765 to 2300 . Climatic Change, 109, 213-241, https://doi.org/10.1007/ s10584-011-0156-z.

Mitchell, D. M., S. M. Osprey, L. J. Gray, N. Butchart, S. C. Hardiman, A. J. Charlton-Perez, and P. Watson, 2012: The effect of climate change on the variability of the Northern Hemisphere stratospheric polar vortex. J. Atmos. Sci., 69, 2608-2618, https://doi.org/10.1175/JAS-D-12-021.1.

Oberländer, S., U. Langematz, and S. Meul, 2013: Unraveling impact factors for future changes in the Brewer-Dobson circulation. J. Geophys. Res. Atmos., 118, 10 296-10312, https:// doi.org/10.1002/jgrd.50775.

Perlwitz, J., and N. Harnik, 2003: Observational evidence of a stratospheric influence on the troposphere by planetary wave reflection. J. Climate, 16, 3011-3026, https://doi.org/10.1175/ 1520-0442(2003)016<3011:OEOASI > 2.0.CO;2.

Plumb, R., 1985: On the three-dimensional propagation of stationary waves. J. Atmos. Sci., 42, 217-229, https://doi.org/ 10.1175/1520-0469(1985)042<0217:OTTDPO > 2.0.CO;2.

Schimanke, S., T. Spangehl, H. Huebener, and U. Cubasch, 2013: Variability and trends of major stratospheric warmings in simulations under constant and increasing GHG concentrations. Climate Dyn., 40, 1733-1747, https://doi.org/10.1007/ s00382-012-1530-x.

Schmidt, H., and Coauthors, 2013: Response of the middle atmosphere to anthropogenic and natural forcings in the CMIP5 simulations with the Max Planck Institute Earth system model. J. Adv. Model. Earth Syst., 5, 98-116, https://doi.org/ 10.1002/jame.20014.

Shaw, T. A., and J. Perlwitz, 2013: The life cycle of Northern Hemisphere downward wave coupling between the stratosphere and troposphere. J. Climate, 26, 1745-1763, https://doi.org/ 10.1175/JCLI-D-12-00251.1.

$\longrightarrow,-$, and N. Harnik, 2010: Downward wave coupling between the stratosphere and troposphere: The importance of meridional wave guiding and comparison with zonal-mean coupling. J. Climate, 23, 6365-6381, https://doi.org/10.1175/ 2010JCLI3804.1. 
, P. A. Newman, and S. Pawson, 2011: The impact of stratospheric ozone changes on downward wave coupling in the Southern Hemisphere. J. Climate, 24, 4210-4229, https:// doi.org/10.1175/2011JCLI4170.1.

,$- \ldots$, and O. Weiner, 2014: Troposphere-stratosphere coupling: Links to North Atlantic weather and climate, including their representation in CMIP5 models. J. Geophys. Res. Atmos., 119, 5864-5880, https://doi.org/10.1002/2013JD021191.

Shepherd, T. G., and C. McLandress, 2011: A robust mechanism for strengthening of the Brewer-Dobson circulation in response to climate change: Critical-layer control of subtropical wave breaking. J. Atmos. Sci., 68, 784-797, https://doi.org/ 10.1175/2010JAS3608.1.

SPARC CCMVal, 2010: SPARC report on the evaluation of chemistry climate models. SPARC Rep. 5, 7 pp.
Tomikawa, Y., 2010: Persistence of easterly wind during major stratospheric sudden warmings. J. Climate, 23, 5258-5267, https://doi.org/10.1175/2010JCLI3507.1.

Tung, K. K., and W. W. Orlando, 2003: The $\mathrm{k}^{-3}$ and $\mathrm{k}^{-5 / 3}$ energy spectrum of atmospheric turbulence: Quasigeostrophic twolevel model simulation. J. Atmos. Sci., 60, 824-835, https:// doi.org/10.1175/1520-0469(2003)060<0824:TKAKES >2.0.CO;2.

Wettstein, J. J., and J. M. Wallace, 2010: Observed patterns of month-to-month storm-track variability and their relationship to the background flow. J. Atmos. Sci., 67, 1420-1437, https:// doi.org/10.1175/2009JAS3194.1.

Woollings, T., J. M. Gregory, J. G. Pinto, M. Reyers, and D. J. Brayshaw, 2012: Response of the North Atlantic storm track to climate change shaped by ocean-atmosphere coupling. Nat. Geosci., 5, 313-317, https://doi.org/10.1038/ngeo1438. 